\title{
Coupling of Two Partial Differential Equations and its Application, II - the Case of Briot-Bouquet Type PDEs
}

\author{
By \\ Hidetoshi TAHARA*
}

\begin{abstract}
Let $F(t, x, u, v)$ be a holomorphic function in a neighborhood of the origin of $\mathbb{C}^{4}$ satisfying $F(0, x, 0,0) \equiv 0$ and $(\partial F / \partial v)(0, x, 0,0) \equiv 0 ;$ then the equation (A) $t \partial u / \partial t=F(t, x, u, \partial u / \partial x)$ is called a Briot-Bouquet type partial differential equation, and the function $\lambda(x)=(\partial F / \partial u)(0, x, 0,0)$ is called the characteristic exponent. This paper considers a reduction of this equation (A) to a simple form (B) $t \partial w / \partial t=\lambda(x) w$ under the assumption $\lambda(0) \notin(-\infty, 0] \cup\{1,2, \ldots\}$. The reduction is done by considering the coupling of two equations (A) and (B), and by solving their coupling equations. The result is applied to the problem of finding all the singular solutions of (A).
\end{abstract}

\section{$\S 1 . \quad$ Introduction}

Let $(t, x)$ be the variables in $\mathbb{C}_{t} \times \mathbb{C}_{x}$, and let $F(t, x, u, v)$ be a holomorphic function defined in a polydisk $\Delta$ centered at the origin of $\mathbb{C}_{t} \times \mathbb{C}_{x} \times \mathbb{C}_{u} \times \mathbb{C}_{v}$. In the previous paper [3], we have established the equivalence of the following two partial differential equations

$$
\frac{\partial u}{\partial t}=F\left(t, x, u, \frac{\partial u}{\partial x}\right) \text { and } \frac{\partial w}{\partial t}=0
$$

Communicated by H. Okamoto. Received June 9, 2008.

2000 Mathematics Subject Classification(s): Primary 35A22; Secondary 35A20, 35C10. Key words: coupling equation, Briot-Bouquet type PDE, equivalence of two PDEs, singular solution, analytic continuation.

This research was partially supported by the Grant-in-Aid for Scientific Research No. 19540197 of Japan Society for the Promotion of Science.

*Department of Mathematics, Sophia University, Kioicho, Chiyoda-ku, Tokyo 102-8554, Japan.

e-mail: h-tahara@hoffman.cc.sophia.ac.jp 
by considering the coupling of these two equations and by solving their coupling equations.

In this paper, we will apply similar arguments to the following nonlinear singular partial differential equation

$$
t \frac{\partial u}{\partial t}=F\left(t, x, u, \frac{\partial u}{\partial x}\right)
$$

under the assumptions

$\left.\mathrm{A}_{1}\right) F(t, x, u, v)$ is holomorphic in $\Delta$,

$\left.\mathrm{A}_{2}\right) F(0, x, 0,0)=0$ in $\Delta_{0}=\Delta \cap\{t=0, u=0, v=0\}$, and

$\left.\mathrm{A}_{3}\right) \frac{\partial F}{\partial v}(0, x, 0,0)=0$ in $\Delta_{0}$.

In the book of Gérard-Tahara [1], the equation (1.1) is called a BriotBouquet type partial differential equation with respect to $t$ if it satisfies the conditions $\mathrm{A}_{1}$ ), $\mathrm{A}_{2}$ ) and $\mathrm{A}_{3}$ ); the function

$$
\lambda(x)=\frac{\partial F}{\partial u}(0, x, 0,0)
$$

is called the characteristic exponent (or the characteristic exponent function) of (1.1). About the structure of holomorphic and singular solutions of (1.1) in a neighborhood of $(0,0) \in \mathbb{C}_{t} \times \mathbb{C}_{x}$, one can refer to Gérard-Tahara [1], [2] and Yamazawa [4]. Among them, the following theorem is the most fundamental result:

Theorem 1.1 ([2]). If $\lambda(0) \notin\{1,2, \ldots\}$ holds, the equation (1.1) has a unique holomorphic solution $u_{0}(t, x)$ in a neighborhood of $(0,0) \in \mathbb{C}_{t} \times \mathbb{C}_{x}$ satisfying $u_{0}(0, x) \equiv 0$ near $x=0$.

In this paper, from the standpoint of the transformation theory of partial differential equations we will consider the following problem:

Problem 1.2. Find a normal form of the equation (1.1) by considering the coupling of two partial differential equations.

As is seen in the case of Briot-Bouquet's ordinary differential equations (in chapter 4 of [1]), it will be reasonable to treat the equation

$$
t \frac{\partial w}{\partial t}=\lambda(x) w
$$


as a candidate of the normal form of (1.1) in a generic case. In order to justify this assertion, it is enough to discuss their coupling equations

$$
\begin{aligned}
& t \frac{\partial \phi}{\partial t}+\sum_{m \geq 0} D^{m}[F]\left(t, x, u_{0}, \ldots, u_{m+1}\right) \frac{\partial \phi}{\partial u_{m}}=\lambda(x) \phi, \quad \text { and } \\
& t \frac{\partial \psi}{\partial t}+\sum_{m \geq 0}\left(\sum_{0 \leq i \leq m} \lambda_{m, i}(x) w_{i}\right) \frac{\partial \psi}{\partial w_{m}}=F(t, x, \psi, D[\psi]),
\end{aligned}
$$

where $D$ is the vector field with infinitely many variables $\left(x, u_{0}, u_{1}, \ldots\right)$ (resp. $\left.\left(x, w_{0}, w_{1}, \ldots\right)\right)$ :

$$
D=\frac{\partial}{\partial x}+\sum_{i \geq 0} u_{i+1} \frac{\partial}{\partial u_{i}} \quad\left(\text { resp. } D=\frac{\partial}{\partial x}+\sum_{i \geq 0} w_{i+1} \frac{\partial}{\partial w_{i}}\right)
$$

and

$$
\lambda_{m, i}(x)=\frac{m !}{i !(m-i) !}\left(\frac{\partial}{\partial x}\right)^{m-i} \lambda(x), \quad 0 \leq i \leq m .
$$

In the equation $(\Phi)($ resp. $(\Psi)),\left(t, x, u_{0}, u_{1}, \ldots\right) \in \mathbb{C}_{t} \times \mathbb{C}_{x} \times \mathbb{C}_{u}^{\infty}$ (resp. $\left(t, x, w_{0}\right.$, $\left.\left.w_{1}, \ldots\right) \in \mathbb{C}_{t} \times \mathbb{C}_{x} \times \mathbb{C}_{w}^{\infty}\right)$ are infinitely many complex variables, and $\phi=$ $\phi\left(t, x, u_{0}, u_{1}, \ldots\right)$ (resp. $\psi=\psi\left(t, x, w_{0}, w_{1}, \ldots\right)$ ) is the unknown function. Roughly speaking, the role of these equations is explained as

Proposition 1.3. Suppose the conditions $\left.\left.\mathrm{A}_{1}\right), \mathrm{A}_{2}\right), \mathrm{A}_{3}$ ) and that

$$
\begin{aligned}
& |i+\lambda(x)(j-1)| \geq \sigma(i+j) \quad \text { on } D_{R}=\{x \in \mathbb{C} ;|x| \leq R\} \\
& \quad \text { for any }(i, j) \in \mathbb{N} \times \mathbb{N} \backslash\{(0,0),(0,1)\}
\end{aligned}
$$

for some $\sigma>0$ and $R>0$. Then, we have the following results.

(1) The equation $(\Phi)$ (resp. $(\Psi)$ ) has a holomorphic solution $\phi=\phi(t, x$, $\left.u_{0}, u_{1}, \ldots\right)\left(\right.$ resp. $\left.\psi=\psi\left(t, x, u_{0}, u_{1}, \ldots\right)\right)$ in a suitable domain in $\mathbb{C}_{t} \times \mathbb{C}_{x} \times \mathbb{C}_{u}^{\infty}$ (resp. $\left.\mathbb{C}_{t} \times \mathbb{C}_{x} \times \mathbb{C}_{w}^{\infty}\right)$.

(2) If $u(t, x)$ (resp. $w(t, x))$ is a solution of (1.1) (resp. (1.3)), then the function $w(t, x)=\phi(t, x, u, \partial u / \partial x, \ldots)(\operatorname{resp} . u(t, x)=\psi(t, x, w, \partial w / \partial x, \ldots))$ is a solution of (1.3) (resp. (1.1)).

(3) The two transformations $u(t, x) \mapsto w(t, x)=\phi(t, x, u, \partial u / \partial x, \ldots)$ and $w(t, x) \mapsto u(t, x)=\psi(t, x, w, \partial w / \partial x, \ldots)$ are invertible, and one is the inverse of the other.

Hence, we have the following main theorem of this paper.

Theorem 1.4 (Main theorem). Suppose the conditions $\left.\left.\mathrm{A}_{1}\right), \mathrm{A}_{2}\right), \mathrm{A}_{3}$ ) and (1.4) (for some $\sigma>0$ and $R>0$ ). Then the following two equations are 
equivalent:

$$
t \frac{\partial u}{\partial t}=F\left(t, x, u, \frac{\partial u}{\partial x}\right) \quad \text { and } \quad t \frac{\partial w}{\partial t}=\lambda(x) w
$$

The meaning of the equivalence of two equations will be defined later (see Definition 2.7). We have used the notation: $\mathbb{N}=\{0,1,2, \ldots\}$.

The paper is organized as follows. In the next section 2 , we will present some basic results in the formal theory of the coupling of two partial differential equations (developed in [3]). In section 3, we will prove Theorem 1.4 by the following steps: in subsections 3.1 and 3.2 we will find formal solutions of the coupling equations $(\Phi)$ and $(\Psi)$ : this shows the formal equivalence of the two partial differential equations in (1.5), in subsections 3.3 and 3.4 we will prove the convergence of the formal solutions, and in subsection 3.5 we will establish the equivalence of the two equations in (1.5) in a concrete sense. In the last section 4 , by using our main theorem (Theorem 1.4) we will determine all the singular solutions of (1.1) in a neighborhood of $(0,0) \in \mathbb{C}_{t} \times \mathbb{C}_{x}$.

\section{$\S 2 . \quad$ Basics on the Coupling Equations}

In this section, we will survey basic results in the formal theory of the coupling of two singular partial differential equations

$$
t \frac{\partial u}{\partial t}=F\left(t, x, u, \frac{\partial u}{\partial x}\right)
$$

(where $(t, x) \in \mathbb{C}_{t} \times \mathbb{C}_{x}$ are variables and $u=u(t, x)$ is the unknown function) and

$$
t \frac{\partial w}{\partial t}=G\left(t, x, w, \frac{\partial w}{\partial x}\right)
$$

(where $(t, x) \in \mathbb{C}_{t} \times \mathbb{C}_{x}$ are variables and $w=w(t, x)$ is the unknown function). For simplicity we suppose that $F\left(t, x, u_{0}, u_{1}\right)$ (resp. $\left.G\left(t, x, w_{0}, w_{1}\right)\right)$ is a holomorphic function defined in a neighborhood of the origin of $\mathbb{C}_{t} \times \mathbb{C}_{x} \times \mathbb{C}_{u_{0}} \times \mathbb{C}_{u_{1}}$ (resp. $\mathbb{C}_{t} \times \mathbb{C}_{x} \times \mathbb{C}_{w_{0}} \times \mathbb{C}_{w_{1}}$ ).

For a function $\phi=\phi\left(t, x, u_{0}, u_{1}, \ldots\right)$ with infinitely many variables $(t, x$, $\left.u_{0}, u_{1}, \ldots\right)$ we define $D[\phi]\left(t, x, u_{0}, u_{1}, \ldots\right)$ by

$$
D[\phi]=\frac{\partial \phi}{\partial x}\left(t, x, u_{0}, u_{1}, \ldots\right)+\sum_{i \geq 0} u_{i+1} \frac{\partial \phi}{\partial u_{i}}\left(t, x, u_{0}, u_{1}, \ldots\right) .
$$


For $m \geq 2$ we define $D^{m}[\phi]$ as follows: $D^{2}[\phi]=D[D[\phi]], D^{3}[\phi]=D\left[D^{2}[\phi]\right]$, and so on. If $\phi$ is a function with $(p+3)$-variables $\left(t, x, u_{0}, \ldots, u_{p}\right)$ then $D^{m}[\phi]$ is a function with $(p+m+3)$-variables $\left(t, x, u_{0}, \ldots, u_{p+m}\right)$. It is clear that

$$
D^{m}[\phi]\left(t, x, u, \frac{\partial u}{\partial x}, \ldots\right)=\left(\frac{\partial}{\partial x}\right)^{m}\left[\phi\left(t, x, u, \frac{\partial u}{\partial x}, \ldots\right)\right]
$$

holds for any $m \in \mathbb{N}$ and any function $u(t, x)$.

Of course, if $\psi=\psi\left(t, x, w_{0}, w_{1}, \ldots\right)$ is a function with variables $\left(t, x, w_{0}\right.$, $\left.w_{1}, \ldots\right)$ the notation $D[\psi]\left(t, x, w_{0}, w_{1}, \ldots\right)$ is read as

$$
D[\psi]=\frac{\partial \psi}{\partial x}\left(t, x, w_{0}, w_{1}, \ldots\right)+\sum_{i \geq 0} w_{i+1} \frac{\partial \psi}{\partial w_{i}}\left(t, x, w_{0}, w_{1}, \ldots\right) .
$$

We can regard $D$ as a vector field with infinitely many variables $\left(x, u_{0}, u_{1}, \ldots\right)$ (resp. $\left.\left(x, w_{0}, w_{1}, \ldots\right)\right)$ :

$$
D=\frac{\partial}{\partial x}+\sum_{i \geq 0} u_{i+1} \frac{\partial}{\partial u_{i}} \quad\left(\text { resp. } D=\frac{\partial}{\partial x}+\sum_{i \geq 0} w_{i+1} \frac{\partial}{\partial w_{i}}\right) .
$$

Usually, this operator $D$ is called the totally derivative operator.

Definition 2.1. The coupling of two partial differential equations (A) and (B) means that we consider the following partial differential equation with infinitely many variables $\left(t, x, u_{0}, u_{1}, \ldots\right)$

(Ф) $\quad t \frac{\partial \phi}{\partial t}+\sum_{m \geq 0} D^{m}[F]\left(t, x, u_{0}, \ldots, u_{m+1}\right) \frac{\partial \phi}{\partial u_{m}}=G(t, x, \phi, D[\phi])$

(where $\phi=\phi\left(t, x, u_{0}, u_{1}, \ldots\right)$ is the unknown function), or the following partial differential equation with infinitely many variables $\left(t, x, w_{0}, w_{1}, \ldots\right)$

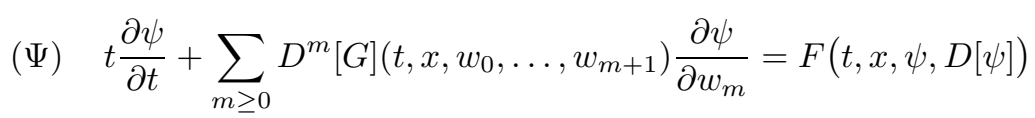

(where $\psi=\psi\left(t, x, w_{0}, w_{1}, \ldots\right)$ is the unknown function).

\section{§2.1. The formal meaning of the coupling equation}

First, let us explain the meaning of the coupling equations $(\Phi)$ and $(\Psi)$ in the formal sense. Here, "in the formal sense" means that the result is true if the formal calculation makes sense. For simplicity we write

$$
\phi(t, x, u, \partial u / \partial x, \ldots)=\phi\left(t, x, u, \frac{\partial u}{\partial x}, \frac{\partial^{2} u}{\partial x^{2}}, \ldots, \frac{\partial^{n} u}{\partial x^{n}}, \ldots\right) .
$$

The convenience of considering the coupling equation lies in the following proposition. 
Proposition 2.2. (1) If $\phi\left(t, x, u_{0}, u_{1}, \ldots\right)$ is a solution of $(\Phi)$ and if $u(t, x)$ is a solution of $(\mathrm{A})$, then the function $w(t, x)=\phi(t, x, u, \partial u / \partial x, \ldots)$ is a solution of $(\mathrm{B})$.

(2) If $\psi\left(t, x, w_{0}, w_{1}, \ldots\right)$ is a solution of $(\Psi)$ and if $w(t, x)$ is a solution of (B), then the function $u(t, x)=\psi(t, x, w, \partial w / \partial x, \ldots)$ is a solution of $(\mathrm{A})$.

Proof. This result corresponds to [Proposition 3.1.1 in [3]], the proof of which works also in this case; we have only to replace $\partial / \partial t$ by $t \partial / \partial t$.

In order to state the relation between $(\Phi)$ and $(\Psi)$, we need the following notion of the reversibility of $\phi\left(t, x, u_{0}, u_{1}, \ldots\right)$.

Definition 2.3. Let $\phi\left(t, x, u_{0}, u_{1}, \ldots\right)$ be a function of $\left(t, x, u_{0}, u_{1}, \ldots\right)$. We say that the relation $w=\phi(t, x, u, \partial u / \partial x, \ldots)$ is reversible with respect to $u$ and $w$ if there is a function $\psi\left(t, x, w_{0}, w_{1}, \ldots\right)$ of $\left(t, x, w_{0}, w_{1}, \ldots\right)$ such that the relation

$$
\left\{\begin{aligned}
w_{0}= & \phi\left(t, x, u_{0}, u_{1}, u_{2}, \ldots\right) \\
w_{1}= & D[\phi]\left(t, x, u_{0}, u_{1}, u_{2}, \ldots\right) \\
w_{2}= & D^{2}[\phi]\left(t, x, u_{0}, u_{1}, u_{2}, \ldots\right) \\
& \ldots \cdots \\
& \cdots \cdots
\end{aligned}\right.
$$

is equivalent to

$$
\left\{\begin{aligned}
u_{0}= & \psi\left(t, x, w_{0}, w_{1}, w_{2}, \ldots\right) \\
u_{1}= & D[\psi]\left(t, x, w_{0}, w_{1}, w_{2}, \ldots\right) \\
u_{2}= & D^{2}[\psi]\left(t, x, w_{0}, w_{1}, w_{2}, \ldots\right), \\
& \cdots \cdots \\
& \cdots \cdots
\end{aligned}\right.
$$

In this case the function $\psi\left(t, x, w_{0}, w_{1}, \ldots\right)$ is called the reverse function of $\phi\left(t, x, u_{0}, u_{1}, \ldots\right)$.

By the definition, we see that the reverse function $\psi\left(t, x, w_{0}, w_{1}, \ldots\right)$ of $\phi\left(t, x, u_{0}, u_{1}, \ldots\right)$ is unique, if it exists.

The following proposition gives the relation between two coupling equations $(\Phi)$ and $(\Psi)$ : we can say that the equation $(\Psi)$ is the reverse of $(\Phi)$.

Proposition 2.4. If $\phi\left(t, x, u_{0}, u_{1}, \ldots\right)$ is a solution of $(\Phi)$ and if the relation $w=\phi(t, x, u, \partial u / \partial x, \ldots)$ is reversible with respect to $u$ and $w$, then the reverse function $\psi\left(t, x, w_{0}, w_{1}, \ldots\right)$ is a solution of $(\Psi)$. 
Proof. This result corresponds to [Proposition 3.1.4 in [3]], the proof of which works also in this case; we have only to replace $\partial / \partial t$ by $t \partial / \partial t$.

Recall that the following lemma played an important role in the proof of Proposition 2.4:

Lemma 2.5 ((2) of Lemma 3.1.5 in [3]). Let $\phi\left(t, x, u_{0}, u_{1}, \ldots\right)$ be a function with variables $\left(t, x, u_{0}, u_{1}, \ldots\right)$ and let $K\left(t, x, w_{0}, w_{1}, \ldots\right)$ be a function with variables $\left(t, x, w_{0}, w_{1}, \ldots\right)$. Then, for any $m=0,1,2, \ldots$ we have

$$
D^{m}[K(t, x, \phi, D[\phi], \ldots)]=\left(D^{m}[K]\right)(t, x, \phi, D[\phi], \ldots)
$$

as a function with respect to the variables $\left(t, x, u_{0}, u_{1}, \ldots\right)$.

\section{§2.2. Equivalence of (A) and (B)}

Let $\mathcal{F}$ and $\mathcal{G}$ be function-spaces in which we can consider the following two partial differential equations:

$$
\begin{aligned}
& t \frac{\partial u}{\partial t}=F\left(t, x, u, \frac{\partial u}{\partial x}\right) \quad \text { in } \mathcal{F}, \\
& t \frac{\partial w}{\partial t}=G\left(t, x, w, \frac{\partial w}{\partial x}\right) \quad \text { in } \mathcal{G} .
\end{aligned}
$$

Set

$$
\begin{aligned}
& \mathcal{S}_{A}=\text { the set of all solutions of }[\mathrm{A}] \text { in } \mathcal{F}, \\
& \mathcal{S}_{B}=\text { the set of all solutions of }[\mathrm{B}] \text { in } \mathcal{G} .
\end{aligned}
$$

Then, if the coupling equation $(\Phi)$ has a solution $\phi\left(t, x, u_{0}, u_{1} \ldots\right)$ and if $\phi(t, x$, $u, \partial u / \partial x, \ldots) \in \mathcal{G}$ is well-defined for any $u \in \mathcal{S}_{A}$, we can define the mapping

$$
\Phi: \mathcal{S}_{A} \ni u(t, x) \longmapsto w(t, x)=\phi(t, x, u, \partial u / \partial x, \ldots) \in \mathcal{S}_{B}
$$

If the relation $w=\phi(t, x, u, \partial u / \partial x, \ldots)$ is reversible with respect to $u$ and $w$, and if the reverse function $\psi\left(t, x, w_{0}, w_{1}, \ldots\right)$ satisfies $\psi(t, x, w, \partial w / \partial x, \ldots) \in \mathcal{F}$ for any $w \in \mathcal{S}_{B}$, we can also define the mapping

$$
\Psi: \mathcal{S}_{B} \ni w(t, x) \longmapsto u(t, x)=\psi(t, x, w, \partial w / \partial x, \ldots) \in \mathcal{S}_{A} .
$$

Set $w(t, x)=\phi(t, x, u, \partial u / \partial x, \ldots)$; then by the definition of $D$ we have

$$
\left\{\begin{array}{l}
w=\phi(t, x, u, \partial u / \partial x, \ldots) \\
\partial w / \partial x=D[\phi](t, x, u, \partial u / \partial x, \ldots) \\
\partial^{2} w / \partial x^{2}=D^{2}[\phi](t, x, u, \partial u / \partial x, \ldots) \\
\ldots \ldots \\
\cdots \cdots
\end{array}\right.
$$


Similarly, if we set $u(t, x)=\psi(t, x, w, \partial w / \partial x, \ldots)$ we have

$$
\left\{\begin{array}{l}
u=\phi(t, x, w, \partial w / \partial x, \ldots) \\
\partial u / \partial x=D[\phi](t, x, w, \partial w / \partial x, \ldots), \\
\partial^{2} u / \partial x^{2}=D^{2}[\phi](t, x, w, \partial w / \partial x, \ldots), \\
\quad \cdots . \\
\quad \ldots .
\end{array}\right.
$$

Since (2.1) is equivalent to (2.2) we have the equivalence between (2.5) and (2.6); therefore we have $\Psi \circ \Phi=$ identity in $\mathcal{S}_{A}$ and $\Phi \circ \Psi=$ identity in $\mathcal{S}_{B}$. Thus, we obtain

Theorem 2.6. $\quad$ Suppose that the coupling equation $(\Phi)$ has a solution $\phi\left(t, x, u_{0}, u_{1} \ldots\right)$ and that the relation $w=\phi(t, x, u, \partial u / \partial x, \ldots)$ is reversible with respect to $u$ and $w$. If both mappings (2.3) and (2.4) are well-defined, we can conclude that the both mappings are bijective and one is the inverse of the other.

By this theorem, we may say:

Definition 2.7. (1) If the coupling equation $(\Phi)$ (resp. $(\Psi)$ ) has a solution $\phi\left(t, x, u_{0}, u_{1} \ldots\right)\left(\operatorname{resp} . \psi\left(t, x, w_{0}, w_{1} \ldots\right)\right)$ and if the relation $w=\phi(t, x, u$, $\partial u / \partial x, \ldots)($ resp. $u=\psi(t, x, w, \partial w / \partial x, \ldots))$ is reversible with respect to $u$ and $w$ (or $w$ and $u$ ), then we say that the two equations (A) and (B) are formally equivalent.

(2) In addition, if both mappings (2.3) and (2.4) are well-defined, we say that the two equations $[\mathrm{A}]$ and $[\mathrm{B}]$ are equivalent.

\section{§2.3. A sufficient condition for the reversibility}

As is seen above, the condition of the reversibility of $\phi\left(t, x, u_{0}, u_{1}, \ldots\right)$ is very important. In [Proposition 3.3.1 in [3]] we gave one sufficient condition for the relation $w=\phi(t, x, u, \partial u / \partial x, \ldots)$ to be reversible with respect to $u$ and $w$. In this section, we will give another sufficient condition.

Let us introduce the notations: $D_{R}=\{x \in \mathbb{C} ;|x| \leq R\}, \mathcal{O}_{R}$ denotes the ring of holomorphic functions in a neighborhood of $D_{R}$, and $\mathcal{H}_{k, R}\left[t, u_{0}, \ldots, u_{p}\right]$ denotes the set of all homogeneous polynomials of degree $k$ in $\left(t, u_{0}, \ldots, u_{p}\right)$ with coefficients in $\mathcal{O}_{R}$.

Proposition 2.8. Let $\phi\left(t, x, u_{0}, u_{1}, \ldots\right)$ be of the form

$$
\phi=\sum_{k \geq 1} \phi_{k}\left(t, x, u_{0}, \ldots, u_{k-1}\right) \in \sum_{k \geq 1} \mathcal{H}_{k, R}\left[t, u_{0}, \ldots, u_{k-1}\right] .
$$


If $\left(\partial \phi_{1} / \partial u_{0}\right) \neq 0$ on $D_{R}$, the relation $w=\phi(t, x, u, \partial u / \partial x, \ldots)$ is reversible with respect to $u$ and $w$, and the reverse function $\psi\left(t, x, w_{0}, w_{1}, \ldots\right)$ is also of the form

$$
\psi=\sum_{k \geq 1} \psi_{k}\left(t, x, w_{0}, \ldots, w_{k-1}\right) \in \sum_{k \geq 1} \mathcal{H}_{k, R}\left[t, w_{0}, \ldots, w_{k-1}\right]
$$

with $\left(\partial \psi_{1} / \partial w_{0}\right) \neq 0$ on $D_{R}$.

To prove this, let us consider the following equation with respect to the unknown function $\psi=\psi\left(t, x, w_{0}, w_{1}, \ldots\right)$ :

$$
w_{0}=\phi\left(t, x, \psi, D[\psi], D^{2}[\psi], \ldots\right) \quad \text { in } \sum_{k \geq 1} \mathcal{H}_{k, R}\left[t, w_{0}, \ldots, w_{k-1}\right] .
$$

We have

Lemma 2.9. Let $\phi\left(t, x, u_{0}, u_{1}, \ldots\right)$ be of the form (2.7), and suppose that $\left(\partial \phi_{1} / \partial u_{0}\right) \neq 0$ holds on $D_{R}$. Then the equation (2.9) has a unique solution $\psi\left(t, x, w_{0}, w_{1}, \ldots\right)$ of the form (2.8) with $\left(\partial \psi_{1} / \partial w_{0}\right) \neq 0$ on $D_{R}$, and it satisfies also

$$
u_{0}=\psi\left(t, x, \phi, D[\phi], D^{2}[\phi], \ldots\right) \quad \text { in } \sum_{k \geq 1} \mathcal{H}_{k, R}\left[t, u_{0}, \ldots, u_{k-1}\right] .
$$

Proof. For simplicity we set

$$
\mathcal{M}^{p}=\sum_{k \geq p} \mathcal{H}_{k, R}\left[t, w_{0}, \ldots, w_{k-1}\right], \quad p=1,2, \ldots
$$

Set $\phi_{1}=\alpha(x) t+\beta(x) u_{0} \in \mathcal{H}_{1, R}\left[t, u_{0}\right]$; by the assumption we have $\beta(x) \neq 0$ on $D_{R}$. Then our equation (2.9) is written as

$$
w_{0}=\alpha(x) t+\beta(x) \psi+\sum_{k \geq 2} \phi_{k}\left(t, x, \psi, \ldots, D^{k-1}[\psi]\right) \quad \text { in } \mathcal{M}^{1} .
$$

Let

$$
\psi=\sum_{k \geq 1} \psi_{k}\left(t, x, w_{0}, \ldots, w_{k-1}\right) \in \mathcal{M}^{1}
$$

be the unknown function. By substituting this into the equation (2.11) and by considering the equation modulo $\mathcal{M}^{p+1}$ we have

$$
\begin{aligned}
w_{0} & \equiv \alpha(x) t+\beta(x)\left(\psi_{1}+\cdots+\psi_{p}\right) \\
& +\sum_{k=2}^{p} \phi_{k}\left(t, x, \sum_{j=1}^{p+1-k} \psi_{j}, \ldots, \sum_{j=1}^{p+1-k} D^{k-1}\left[\psi_{j}\right]\right) \quad\left(\bmod \mathcal{M}^{p+1}\right) .
\end{aligned}
$$


In the case $p=1,(2.12)_{1}$ gives the equality

$$
w_{0}=\alpha(x) t+\beta(x) \psi_{1}
$$

and so we have

$$
\psi_{1}=-(\alpha(x) / \beta(x)) t+(1 / \beta(x)) w_{0} \in \mathcal{H}_{1, R}\left[t, w_{0}\right] .
$$

Thus we have seen that $\psi_{1} \in \mathcal{H}_{1, R}\left[t, w_{0}\right]$ is determined uniquely, and it satisfies $\left(\partial \psi_{1} / \partial w_{0}\right) \neq 0$ on $D_{R}$. If we take $p=2$, we have also the equality

$$
w_{0}=\alpha(x) t+\beta(x)\left(\psi_{1}+\psi_{2}\right)+\phi_{2}\left(t, x, \psi_{1}, D\left[\psi_{1}\right]\right)
$$

where $\psi_{1}=-(\alpha(x) / \beta(x)) t+(1 / \beta(x)) w_{0}$ and so $D\left[\psi_{1}\right]=-(\alpha(x) / \beta(x))^{\prime} t+$ $(1 / \beta(x))^{\prime} w_{0}+(1 / \beta(x)) w_{1}$. Since $(2.13)$ is true, we have

$$
\psi_{2}=-(1 / \beta(x)) \phi_{2}\left(t, x, \psi_{1}, D\left[\psi_{1}\right]\right) \in \mathcal{H}_{2, R}\left[t, w_{0}, w_{1}\right] ;
$$

this proves that $\psi_{2} \in \mathcal{H}_{2, R}\left[t, w_{0}, w_{1}\right]$ is also determined uniquely.

For a general $p \geq 3$, by (2.13) and $(2.12)_{p}$ we have

$$
\begin{aligned}
(2.14)_{p} & \sum_{k=2}^{p} \psi_{k}\left(t, x, w_{0}, \ldots, w_{k-1}\right) \\
& \equiv-\frac{1}{\beta(x)} \sum_{k=2}^{p} \phi_{k}\left(t, x, \sum_{j=1}^{p+1-k} \psi_{j}, \ldots, \sum_{j=1}^{p+1-k} D^{k-1}\left[\psi_{j}\right]\right) \quad\left(\bmod \mathcal{M}^{p+1}\right) .
\end{aligned}
$$

Since the right-hand side of $(2.14)_{p}$ is determined only by $\psi_{1}, \ldots, \psi_{p-1}$, the formula $(2.14)_{p}$ guarantees that $\psi_{p} \in \mathcal{H}_{p, R}\left[t, w_{0}, \ldots, w_{p-1}\right]$ is determined uniquely if $\psi_{1}, \ldots, \psi_{p-1}$ are known.

Thus, we can conclude that all $\psi_{p} \in \mathcal{H}_{p, R}\left[t, w_{0}, \ldots, w_{p-1}\right](p=1,2, \ldots)$ are determined uniquely by the formula $(2.14)_{p}$ by induction on $p$. This proves the former half of Lemma 2.9.

Next let us show the latter half of this lemma: to do so it is sufficient to prove the following equality

$$
u_{0} \equiv \sum_{k=1}^{p} \psi_{k}\left(t, x, \sum_{h=1}^{p} \phi_{h}, \ldots, \sum_{h=1}^{p} D^{k-1}\left[\phi_{h}\right]\right) \quad\left(\bmod \mathcal{M}_{u}^{p+1}\right)
$$

for all $p=1,2, \ldots$, where $\mathcal{M}_{u}^{p+1}$ is the same one as $\mathcal{M}^{p+1}$ with $\left(w_{0}, w_{1}, \ldots\right)$ replaced by $\left(u_{0}, u_{1}, \ldots\right)$. In the case $p=1$ we have

$$
\begin{aligned}
\psi_{1}\left(t, x, \phi_{1}\right) & =-(\alpha(x) / \beta(x)) t+(1 / \beta(x)) \phi_{1} \\
& =-(\alpha(x) / \beta(x)) t+(1 / \beta(x))\left(\alpha(x) t+\beta(x) u_{0}\right)=u_{0},
\end{aligned}
$$


which proves $(2.15)_{1}$.

Let $p \geq 2$ and suppose that $(2.15)_{p-1}$ is already proved. Then, by Lemma 2.5 and the fact $D^{j}\left[u_{0}\right]=u_{j}$ we have

$$
u_{j} \equiv \sum_{k=1}^{p-1} D^{j}\left[\psi_{k}\right]\left(t, x, \sum_{h=1}^{p-1} \phi_{h}, \ldots, \sum_{h=1}^{p-1} D^{k-1+j}\left[\phi_{h}\right]\right) \quad\left(\bmod \mathcal{M}_{u}^{p}\right)
$$

for $j=0,1,2, \ldots$. Since this equality is considered modulo $\mathcal{M}_{u}^{p}$, this implies that

$$
u_{j} \equiv \sum_{k=1}^{p-1} D^{j}\left[\psi_{k}\right]\left(t, x, \sum_{h=1}^{p} \phi_{h}, \ldots, \sum_{h=1}^{p} D^{k-1+j}\left[\phi_{h}\right]\right) \quad\left(\bmod \mathcal{M}_{u}^{p}\right)
$$

holds for all $j=0,1,2, \ldots$. Since $\psi_{1}, \ldots, \psi_{p}$ are determined so that the relation $(2.12)_{p}$ holds, we have

$$
\begin{aligned}
w_{0} \equiv & \alpha(x) t+\beta(x)\left(\psi_{1}+\cdots+\psi_{p}\right) \\
& +\sum_{k=2}^{p} \phi_{k}\left(t, x, \sum_{j=1}^{p-1} \psi_{j}, \ldots, \sum_{j=1}^{p-1} D^{k-1}\left[\psi_{j}\right]\right) \quad\left(\bmod \mathcal{M}^{p+1}\right) .
\end{aligned}
$$

By substituting

$$
w_{i}=\sum_{h=1}^{p} D^{i}\left[\phi_{h}\right]\left(t, x, u_{0}, \ldots, u_{h-1+i}\right), \quad i=0,1,2, \ldots
$$

into the both sides of (2.17), we have the relation

$$
\begin{aligned}
& \sum_{h=1}^{p} \phi_{h}\left(t, x, u_{0}, \ldots, u_{h-1}\right) \\
& \equiv \alpha(x) t+\beta(x) \sum_{j=1}^{p} \psi_{j}\left(t, x, \sum_{h=1}^{p} \phi_{h}, \ldots, \sum_{h=1}^{p} D^{j-1}\left[\phi_{h}\right]\right) \\
& +\sum_{k=2}^{p} \phi_{k}\left(t, x, \sum_{j=1}^{p-1} \psi_{j}\left(t, x, \sum_{h=1}^{p} \phi_{h}, \ldots, \sum_{h=1}^{p} D^{j-1}\left[\phi_{h}\right]\right), \ldots,\right. \\
& \left.\sum_{j=1}^{p-1} D^{k-1}\left[\psi_{j}\right]\left(t, x, \sum_{h=1}^{p} \phi_{h}, \ldots, \sum_{h=1}^{p} D^{j-1+k-1}\left[\phi_{h}\right]\right)\right)
\end{aligned}
$$

$\left(\bmod \mathcal{M}_{u}^{p+1}\right)$ 
as functions with respect to the variables $\left(t, x, u_{0}, u_{1}, \ldots\right)$. Therefore, by applying (2.16) to the right-hand side of (2.18) we obtain

$$
\begin{aligned}
& \sum_{h=1}^{p} \phi_{h}\left(t, x, u_{0}, \ldots, u_{h-1}\right) \\
& \equiv \alpha(x) t+\beta(x) \sum_{j=1}^{p} \psi_{j}\left(t, x, \sum_{h=1}^{p} \phi_{h}, \ldots, \sum_{h=1}^{p} D^{j-1}\left[\phi_{h}\right]\right) \\
& \quad+\sum_{k=2}^{p} \phi_{k}\left(t, x, u_{0}, \ldots, u_{k-1}\right) \quad\left(\bmod \mathcal{M}_{u}^{p+1}\right),
\end{aligned}
$$

and so by (2.19) we have

$$
\begin{aligned}
& \phi_{1}\left(t, x, u_{0}\right) \\
& \equiv \alpha(x) t+\beta(x) \sum_{j=1}^{p} \psi_{j}\left(t, x, \sum_{h=1}^{p} \phi_{h}, \ldots, \sum_{h=1}^{p} D^{k}\left[\phi_{h}\right]\right) \quad\left(\bmod \mathcal{M}_{u}^{p+1}\right) .
\end{aligned}
$$

Thus, by applying $\phi_{1}=\alpha(x) t+\beta(x) u_{0}$ and by using the condition $\beta(x) \neq 0$ on $D_{R}$ we obtain

$$
u_{0} \equiv \sum_{j=1}^{p} \psi_{j}\left(t, x, \sum_{h=1}^{p} \phi_{h}, \ldots, \sum_{h=1}^{p} D^{k}\left[\phi_{h}\right]\right) \quad\left(\bmod \mathcal{M}_{u}^{p+1}\right) .
$$

This proves $(2.15)_{p}$.

Thus, by induction on $p$ we can prove $(2.15)_{p}$ for all $p=1,2, \ldots$ This proves the latter half of Lemma 2.9 .

Proof of Proposition 2.8. Let $\psi\left(t, x, w_{0}, w_{1}, \ldots\right)$ be the solution of $(2.9)$ in Lemma 2.9. Let us show the equivalence between the relations

$$
u_{j}=D^{j}[\psi]\left(t, x, w_{0}, w_{1}, \ldots\right), \quad j=0,1,2, \ldots
$$

and

$$
w_{j}=D^{j}[\phi]\left(t, x, u_{0}, u_{1}, \ldots\right), \quad j=0,1,2, \ldots
$$

Let us apply $D^{j}$ to the both sides of the equality (2.9); by Lemma 2.5 we have

$$
w_{j}=D^{j}[\phi]\left(t, x, \psi, D[\psi], D^{2}[\psi], \ldots\right), \quad j=0,1,2, \ldots
$$

and therefore under the relation (2.20) we can get the relation (2.21). Similarly, by applying $D^{j}$ to the both sides of the equality (2.10) we have

$$
u_{j}=D^{j}[\psi]\left(t, x, \phi, D[\phi], D^{2}[\phi], \ldots\right), \quad j=0,1,2, \ldots
$$


and therefore under the relation (2.21) we can get the relation (2.20). This proves Proposition 2.8.

\section{$\S 3 . \quad$ Proof of the Main Theorem}

In this section, we will give a proof of Theorem 1.4 (Main theorem) in the introduction. Let us recall our situation again.

Let $(t, x) \in \mathbb{C}_{t} \times \mathbb{C}_{x}$ be the variables, and let $F(t, x, u, v)$ be a function defined in a polydisk $\Delta$ centered at the origin of $\mathbb{C}_{t} \times \mathbb{C}_{x} \times \mathbb{C}_{u} \times \mathbb{C}_{v}$ satisfying

$\left.\mathrm{A}_{1}\right) F(t, x, u, v)$ is holomorphic in $\Delta$,

A $) F(0, x, 0,0)=0$ in $\Delta_{0}=\Delta \cap\{t=0, u=0, v=0\}$, and

A $) \frac{\partial F}{\partial v}(0, x, 0,0)=0$ in $\Delta_{0}$.

Under these condition, let us consider the following partial differential equation

$$
t \frac{\partial u}{\partial t}=F\left(t, x, u, \frac{\partial u}{\partial x}\right):
$$

this equation is called a Briot-Bouquet type partial differential equation with respect to $t$ (in [1]), and the function

$$
\lambda(x)=\frac{\partial F}{\partial u}(0, x, 0,0)
$$

is called the characteristic exponent of (3.0.1).

Let us seek for a reduction of the equation (3.0.1) to a simple form. As is seen in the case of Briot-Bouquet's ordinary differential equation (in chapter 4 of [1]), it will be reasonable to treat the equation

$$
t \frac{\partial w}{\partial t}=\lambda(x) w
$$

as a candidate of the reduced form of (3.0.1). In order to justify this assertion, it is enough to discuss the following coupling equation

$$
\begin{aligned}
& t \frac{\partial \phi}{\partial t}+\sum_{m \geq 0} D^{m}[F]\left(t, x, u_{0}, \ldots, u_{m+1}\right) \frac{\partial \phi}{\partial u_{m}}=\lambda(x) \phi, \quad \text { and } \\
& t \frac{\partial \psi}{\partial t}+\sum_{m \geq 0}\left(\sum_{0 \leq i \leq m} \lambda_{m, i}(x) w_{i}\right) \frac{\partial \psi}{\partial w_{m}}=F(t, x, \psi, D[\psi])
\end{aligned}
$$

where

$$
\lambda_{m, i}(x)=\frac{m !}{i !(m-i) !}\left(\frac{\partial}{\partial x}\right)^{m-i} \lambda(x), \quad 0 \leq i \leq m .
$$




\section{$\S 3.1$ Formal solutions of $(\Phi)$}

First, let us look for a formal solution of the coupling equation $(\Phi)$ of the form

$$
\phi=\sum_{k \geq 1} \phi_{k}\left(t, x, u_{0}, \ldots, u_{k-1}\right) \in \sum_{k \geq 1} \mathcal{H}_{k, R}\left[t, u_{0}, \ldots, u_{k-1}\right] .
$$

We have

Proposition 3.1.1. $\quad$ Let $R>0$ be sufficiently small. Suppose the conditions $\left.\left.\mathrm{A}_{1}\right), \mathrm{A}_{2}\right), \mathrm{A}_{3}$ ) and that

$$
\begin{aligned}
& |i+\lambda(x)(j-1)| \neq 0 \text { on } D_{R} \\
& \quad \text { for any }(i, j) \in \mathbb{N} \times \mathbb{N} \backslash\{(0,0),(0,1)\} .
\end{aligned}
$$

Then, the coupling equation $(\Phi)$ has a family of formal solutions of the form

$$
\begin{aligned}
& \phi=\frac{-a(x) \beta(x)}{1-\lambda(x)} t+\beta(x) u_{0}+\sum_{k \geq 2} \phi_{k}\left(t, x, u_{0}, \ldots, u_{k-1}\right) \\
& \text { with } \phi_{k}\left(t, x, u_{0}, \ldots, u_{k-1}\right) \in \mathcal{H}_{k, R}\left[t, u_{0}, \ldots, u_{k-1}\right](k=2,3, \ldots),
\end{aligned}
$$

where $a(x)=(\partial F / \partial t)(0, x, 0,0)$ and $\beta(x) \in \mathcal{O}_{R}$. Here, $\beta(x)$ can be chosen arbitrarily, and the other $\phi_{k}\left(t, x, u_{0}, \ldots, u_{k-1}\right)(k=2,3, \ldots)$ are uniquely determined by $\beta(x)$.

In particular, if we take a function $\beta(x) \in \mathcal{O}_{R}$ so that $\beta(x) \neq 0$ holds on $D_{R}$, by Proposition 2.8 we see that the relation $w=\phi(t, x, u, \partial u / \partial x, \ldots)$ is reversible with respect to $u$ and $w$. Hence we obtain

Theorem 3.1.2. Suppose the conditions $\left.\left.\left.\mathrm{A}_{1}\right), \mathrm{A}_{2}\right), \mathrm{A}_{3}\right)$ and (3.1.1). Then, the equation (3.0.1) is formally equivalent to (3.0.2).

Proof of Proposition 3.1.1. By the conditions $\mathrm{A}_{1}$ ), $\mathrm{A}_{2}$ ) and $\mathrm{A}_{3}$ ) we have the expression

$$
F\left(t, x, u_{0}, u_{1}\right)=a(x) t+\lambda(x) u_{0}+\sum_{i+j+\alpha \geq 2} c_{i, j, \alpha}(x) t^{i} u_{0}{ }^{j} u_{1}^{\alpha}
$$

where $a(x), \lambda(x)$ and $c_{i, j, \alpha}(x)(i+j+\alpha \geq 2)$ are all holomorphic functions in a neighborhood of $D_{R}$. We set

$$
R_{p}\left(t, x, u_{0}, u_{1}\right)=\sum_{i+j+\alpha=p} c_{i, j, \alpha}(x) t^{i} u_{0}{ }^{j} u_{1}{ }^{\alpha} \in \mathcal{H}_{p, R}\left[t, u_{0}, u_{1}\right], \quad p \geq 2 .
$$


Then, we have $F\left(t, x, u_{0}, u_{1}\right)=a(x) t+\lambda(x) u_{0}+\sum_{p \geq 2} R_{p}\left(t, x, u_{0}, u_{1}\right)$ and so

$$
\begin{aligned}
& D^{m}[F]\left(t, x, u_{0}, \ldots, u_{m+1}\right) \\
& =a^{(m)}(x) t+\sum_{0 \leq i \leq m} \lambda_{m, i}(x) u_{i}+\sum_{p \geq 2} D^{m}\left[R_{p}\right]\left(t, x, u_{0}, \ldots, u_{m+1}\right)
\end{aligned}
$$

for any $m \in \mathbb{N}$, where $a^{(m)}(x)=(\partial / \partial x)^{m} a(x)$.

Thus, by substituting (3.1.4) into the coupling equation $(\Phi)$ we see that our coupling equation $(\Phi)$ is written in the form

$$
(\tau-\lambda(x)) \phi=-\sum_{m \geq 0} \sum_{p \geq 2} D^{m}\left[R_{p}\right]\left(t, x, u_{0}, \ldots, u_{m+1}\right) \frac{\partial \phi}{\partial u_{m}}
$$

where

$$
\tau=t \frac{\partial}{\partial t}+\sum_{m \geq 0}\left(a^{(m)}(x) t+\sum_{0 \leq i \leq m} \lambda_{m, i}(x) u_{i}\right) \frac{\partial}{\partial u_{m}}
$$

a vector field with infinitely many variables $\left(t, u_{0}, u_{1}, \ldots\right)$.

Now, let us solve the equation (3.1.5). Let

$$
\phi=\sum_{k \geq 1} \phi_{k}\left(t, x, u_{0}, \ldots, u_{k-1}\right) \in \sum_{k \geq 1} \mathcal{H}_{k, R}\left[t, u_{0}, \ldots, u_{k-1}\right]
$$

be the unknown function. Since $D^{m}\left[R_{p}\right]\left(t, x, u_{0}, \ldots, u_{m+1}\right)$ belongs in the class $\mathcal{H}_{p, R}\left[t, u_{0}, \ldots, u_{m+1}\right]$, by substituting (3.1.6) into (3.1.5) and by comparing the homogeneous part of degree $k$ with respect to $\left(t, u_{0}, \ldots, u_{k-1}\right)$ we see that (3.1.5) is decomposed into the following recurrent formulas:

$$
\left(\tau_{1}-\lambda(x)\right) \phi_{1}=0 \quad \text { in } \mathcal{H}_{1, R}\left[t, u_{0}\right]
$$

and for $k \geq 2$

$$
\begin{aligned}
& \left(\tau_{k}-\lambda(x)\right) \phi_{k} \\
& =-\sum_{1 \leq q \leq k-1} \sum_{0 \leq m \leq q-1} D^{m}\left[R_{k-q+1}\right]\left(t, x, u_{0}, \ldots, u_{m+1}\right) \frac{\partial \phi_{q}}{\partial u_{m}} \\
& \text { in } \mathcal{H}_{k, R}\left[t, u_{0}, \ldots, u_{k-1}\right]
\end{aligned}
$$

where

$$
\tau_{k}=t \frac{\partial}{\partial t}+\sum_{0 \leq m \leq k-1}\left(a^{(m)}(x) t+\sum_{0 \leq i \leq m} \lambda_{m, i}(x) u_{i}\right) \frac{\partial}{\partial u_{m}}, \quad k=1,2, \ldots
$$

Thus, to complete the proof of Proposition 3.1.1 it is enough to show the following lemma: 
Lemma 3.1.3. (1) If $\lambda(x) \neq 1$ on $D_{R}$, the equation $\left(\tau_{1}-\lambda(x)\right) \phi_{1}=0$ has a solution $\phi_{1} \in \mathcal{H}_{1, R}\left[t, u_{0}\right]$ of the form

$$
\phi_{1}=\frac{-a(x) \beta(x)}{1-\lambda(x)} t+\beta(x) u_{0}
$$

and $\beta(x) \in \mathcal{O}_{R}$ can be chosen arbitrarily.

(2) Let $k \geq 2$. If $|i+\lambda(x)(j-1)| \neq 0$ on $D_{R}$ for any $(i, j) \in \mathbb{N} \times \mathbb{N}$ with $i+j=k$, then for any $f_{k} \in \mathcal{H}_{k, R}\left[t, u_{0}, \ldots, u_{k-1}\right]$ the equation $\left(\tau_{k}-\lambda(x)\right) \phi_{k}=f_{k}$ has a unique solution $\phi_{k} \in \mathcal{H}_{k, R}\left[t, u_{0}, \ldots, u_{k-1}\right]$.

Proof. The condition $\phi_{1} \in \mathcal{H}_{1, R}\left[t, u_{0}\right]$ means that $\phi_{1}$ is expressed in the form $\phi_{1}=\alpha(x) t+\beta(x) u_{0}$; therefore by substituting this into $\left(\tau_{1}-\lambda(x)\right) \phi_{1}=0$ we can easily verify the result (1).

Let us show (2). Set

$$
\begin{aligned}
f_{k} & =\sum_{i+|j|=k} f_{i, j}(x) t^{i} u_{0}{ }^{j_{0}} \cdots u_{k-1}{ }^{j_{k-1}}, \\
\phi_{k} & =\sum_{i+|j|=k} \phi_{i, j}(x) t^{i} u_{0}^{j_{0}} \cdots u_{k-1}{ }^{j_{k-1}},
\end{aligned}
$$

where $j=\left(j_{0}, \ldots, j_{k-1}\right)$ and $|j|=j_{0}+\cdots+j_{k-1}$. Then, by using the condition $\lambda_{m, m}(x)=\lambda(x)$ (for all $\left.m=0,1,2, \ldots\right)$ we see that the equation $\left(\tau_{k}-\lambda(x)\right) \phi_{k}=$ $f_{k}$ is equivalent to

$$
\begin{aligned}
& \sum_{i+|j|=k}(i+\lambda(x)(|j|-1)) \phi_{i, j}(x) t^{i} u_{0}^{j_{0}} \cdots u_{k-1}^{j_{k-1}} \\
& =\sum_{i+|j|=k} f_{i, j}(x) t^{i} u_{0}{ }^{j_{0}} \cdots u_{k-1}^{j_{k-1}} \\
& -\sum_{i+|j|=k} \phi_{i, j}(x)\left[\sum_{0 \leq m \leq k-1} a^{(m)}(x) j_{m} t^{i+1} u_{0}^{j_{0}} \cdots u_{m}^{j_{m}-1} \cdots u_{k-1}^{j_{k-1}}\right. \\
& \left.+\sum_{1 \leq m \leq k-1} \sum_{0 \leq i \leq m-1} \lambda_{m, i}(x) j_{m} t^{i} u_{0}^{j_{0}} \cdots u_{i}^{j_{i}+1} \cdots u_{m}^{j_{m}-1} \cdots u_{k-1}{ }^{j_{k-1}}\right] .
\end{aligned}
$$

In order to handle this equation, the following total order relation $\prec$ in $A_{k}=\left\{\left(i, j_{0}, \ldots, j_{k-1}\right) \in \mathbb{N} \times \mathbb{N}^{k} ; i+|j|=k\right\}$ will be very convenient: for $(i, j)=\left(i, j_{0}, \ldots, j_{k-1}\right) \in A_{k}$ and $(p, q)=\left(p, q_{0}, \ldots, q_{k-1}\right) \in A_{k}$ we write $(i, j) \prec(p, q)$ if one of the following conditions is satisfied; 
1) $j_{k-1}<q_{k-1}$,

2) $j_{k-1}=q_{k-1}$ and $j_{k-2}<q_{k-2}$,

3) $j_{k-1}=q_{k-1}, j_{k-2}=q_{k-2}$ and $j_{k-3}<q_{k-3}$,

4) and so on.

Then, by comparing the coefficients of $t^{i} u_{0}{ }^{j_{0}} \cdots u_{k-1}{ }^{j_{k-1}}$ in the both sides of (3.1.9) we have

$$
(i+\lambda(x)(|j|-1)) \phi_{i, j}(x)=f_{i, j}(x)+G_{i, j}\left(\phi_{p, q} ;(p, q) \succ(i, j)\right)
$$

where $G_{i, j}\left(\phi_{p, q} ;(p, q) \succ(i, j)\right)$ is the term determined by $\left\{\phi_{p, q} ;(p, q) \succ(i, j)\right\}$. Moreover we see that $G_{0,(0, \ldots, 0, k)} \equiv 0$ holds.

Thus, by the condition $(i+\lambda(x)(|j|-1)) \neq 0$ on $D_{R}$ we can determine $\phi_{i, j}(x) \in \mathcal{O}_{R}$ inductively on $(i, j) \in A_{k}$ from above with respect to the order $\prec$ in $A_{k}$. This proves the result (2).

By using Lemma 3.1.3 we can solve the equations (3.1.7) and (3.1.8). This completes the proof of Proposition 3.1.1.

Thus, we have proved Theorem 3.1.2 which asserts that the equation (3.0.1) is equivalent to (3.0.2); but it is only in a formal sense. To apply this reduction to the study of (3.0.1) in the holomorphic category, we need to prove the convergence of this formal reduction which will be done in subsections 3.3 and 3.4 .

\section{$\S 3.2$. Formal solutions of $(\Psi)$}

Next, let us consider the second coupling equation $(\Psi)$. We have

Proposition 3.2.1. Let $R>0$ be sufficiently small. Suppose the conditions $\left.\left.\mathrm{A}_{1}\right), \mathrm{A}_{2}\right), \mathrm{A}_{3}$ ) and that

$$
\begin{aligned}
& |i+\lambda(x)(j-1)| \neq 0 \text { on } D_{R} \\
& \quad \text { for any }(i, j) \in \mathbb{N} \times \mathbb{N} \backslash\{(0,0),(0,1)\} .
\end{aligned}
$$

Then, the coupling equation $(\Psi)$ has a family of formal solutions of the form

$$
\begin{aligned}
& \psi=\frac{a(x)}{1-\lambda(x)} t+\beta(x) w_{0}+\sum_{k \geq 2} \psi_{k}\left(t, x, w_{0}, \ldots, w_{k-1}\right) \\
& \text { with } \psi_{k}\left(t, x, w_{0}, \ldots, w_{k-1}\right) \in \mathcal{H}_{k, R}\left[t, w_{0}, \ldots, w_{k-1}\right](k=2,3, \ldots),
\end{aligned}
$$

where $a(x)=(\partial F / \partial t)(0, x, 0,0)$ and $\beta(x) \in \mathcal{O}_{R}$. Here, $\beta(x)$ can be chosen arbitrarily, and the other $\psi_{k}\left(t, x, w_{0}, \ldots, w_{k-1}\right)(k=2,3, \ldots)$ are uniquely determined by $\beta(x)$. 
Proof. As in (3.1.3), we have the expression

$$
F\left(t, x, w_{0}, w_{1}\right)=a(x) t+\lambda(x) w_{0}+\sum_{i+j+\alpha \geq 2} c_{i, j, \alpha}(x) t^{i} w_{0}^{j} w_{1}^{\alpha}
$$

where $a(x), \lambda(x)$ and $c_{i, j, \alpha}(x)(i+j+\alpha \geq 2)$ are all holomorphic functions in a neighborhood of $D_{R}$. Therefore, the coupling equation $(\Psi)$ is expressed in the form

$$
\left(\tau^{*}-\lambda(x)\right) \psi=a(x) t+\sum_{i+j+\alpha \geq 2} c_{i, j, \alpha}(x) t^{i} \psi^{j}(D[\psi])^{\alpha}
$$

where

$$
\tau^{*}=t \frac{\partial}{\partial t}+\sum_{m \geq 0}\left(\sum_{0 \leq i \leq m} \lambda_{m, i}(x) w_{i}\right) \frac{\partial}{\partial w_{m}}
$$

a vector field with infinitely many variables $\left(t, w_{0}, w_{1}, \ldots\right)$, and $\lambda_{m, i}(x)=$ $(m ! / i !(m-i) !)(\partial / \partial x)^{m-i} \lambda(x)(0 \leq i \leq m)$. As in section 3.1 we note that $\lambda_{m, m}(x)=\lambda(x)$ holds for all $m=0,1,2, \ldots$

Let

$$
\psi=\sum_{k \geq 1} \psi_{k}\left(t, x, w_{0}, \ldots, w_{k-1}\right) \in \sum_{k \geq 1} \mathcal{H}_{k, R}\left[t, w_{0}, \ldots, w_{k-1}\right]
$$

be the unknown function. Then, by substituting (3.2.4) into (3.2.3) and by comparing the homogeneous parts of degree $k$ with respect to $\left(t, w_{0}, \ldots, w_{k-1}\right)$ we see that (3.2.3) is decomposed into the following recurrent formulas:

$$
\left(\tau_{1}^{*}-\lambda(x)\right) \psi_{1}=a(x) t \quad \text { in } \mathcal{H}_{1, R}\left[t, w_{0}\right]
$$

and for $k \geq 2$

$$
\begin{array}{r}
\left(\tau_{k}^{*}-\lambda(x)\right) \psi_{k}=\sum_{2 \leq i+j+\alpha \leq k} c_{i, j, \alpha}(x) t^{i}\left[\sum_{|p(j)|+|q(\alpha)|=k-i} \psi_{p_{1}} \times\right. \\
\left.\times \cdots \times \psi_{p_{j}} \times D\left[\psi_{q_{1}}\right] \times \cdots \times D\left[\psi_{q_{\alpha}}\right]\right]
\end{array}
$$

in $\mathcal{H}_{k, R}\left[t, w_{0}, \cdots, w_{k-1}\right]$,

where

$$
\tau_{k}^{*}=t \frac{\partial}{\partial t}+\sum_{0 \leq m \leq k-1}\left(\sum_{0 \leq i \leq m} \lambda_{m, i}(x) w_{i}\right) \frac{\partial}{\partial w_{m}}, k=1,2, \ldots,
$$

$|p(j)|=p_{1}+\cdots+p_{j}$ and $|q(\alpha)|=q_{1}+\cdots+q_{\alpha}$. Thus, to complete the proof of Proposition 3.2.1 it is enough to show the following lemma: 
Lemma 3.2.2. (1) If $\lambda(x) \neq 1$ on $D_{R}$, the equation $\left(\tau_{1}^{*}-\lambda(x)\right) \psi_{1}=$ $a(x) t$ has a solution $\psi_{1} \in \mathcal{H}_{1, R}\left[t, w_{0}\right]$ of the form

$$
\psi_{1}=\frac{a(x)}{1-\lambda(x)} t+\beta(x) w_{0}
$$

and $\beta(x) \in \mathcal{O}_{R}$ can be chosen arbitrarily.

(2) Let $k \geq 2$. If $|i+\lambda(x)(j-1)| \neq 0$ on $D_{R}$ for any $(i, j) \in \mathbb{N} \times \mathbb{N}$ with $i+j=k$, then for any $f_{k} \in \mathcal{H}_{k, R}\left[t, w_{0}, \ldots, w_{k-1}\right]$ the equation $\left(\tau_{k}^{*}-\lambda(x)\right) \psi_{k}=$ $f_{k}$ has a unique solution $\psi_{k} \in \mathcal{H}_{k, R}\left[t, w_{0}, \ldots, w_{k-1}\right]$.

The proof of this lemma is almost the same as that of Lemma 3.1.3 and so we may omit the details. By using this lemma we can solve the equations (3.2.5) and (3.2.6). This completes the proof of Proposition 3.2.1.

Now, let us give a more precise form of the formal solution of $(\Psi)$. For $j=\left(j_{0}, j_{1}, \ldots, j_{k-1}\right) \in \mathbb{N}^{k}$ we write $\langle j\rangle=j_{1}+2 j_{2}+\cdots+(k-1) j_{k-1}$. For any $k \in \mathbb{N}^{*}(=\{1,2,3, \ldots\})$ we set

$$
\begin{aligned}
S_{k}= & \left\{(k,(0, \ldots, 0)) \in \mathbb{N} \times \mathbb{N}^{k}\right\} \\
& \cup\left\{(i, j) \in \mathbb{N} \times \mathbb{N}^{k} ; i+|j|=k,|j| \geq 1, i+2(|j|-1) \geq\langle j\rangle\right\} .
\end{aligned}
$$

We have

Proposition 3.2.3. The term $\psi_{k}\left(t, x, w_{0}, \ldots, w_{k-1}\right)($ for $k \geq 2)$ in (3.2.2) has the form

$$
\psi_{k}=\sum_{(i, j) \in S_{k}} \psi_{i, j}(x) t^{i} w_{0}{ }^{j_{0}} w_{1}^{j_{1}} \cdots w_{k-1}{ }^{j_{k-1}} .
$$

Proof. For $k \in \mathbb{N}^{*}(=\{1,2, \ldots\})$ we denote by $\mathcal{G}_{k}$ the set of all the functions $g_{k}\left(t, x, w_{0}, \ldots, w_{k-1}\right) \in \mathcal{H}_{k, R}\left[t, w_{0}, \ldots, w_{k-1}\right]$ of the form

$$
g_{k}=\sum_{(i, j) \in S_{k}} g_{i, j}(x) t^{i} w_{0}^{j_{0}} w_{1}^{j_{1}} \cdots w_{k-1}{ }^{j_{k-1}} .
$$

Then we have the following properties:

1) $\psi_{1}=\left(a(x) /(1-\lambda(x)) t+\beta(x) w_{0} \in \mathcal{G}_{1}\right.$,

2) $t^{i} \in \mathcal{G}_{i}$ for any $i \in \mathbb{N}^{*}$,

3) $t(\partial / \partial t) \mathcal{G}_{k} \subset \mathcal{G}_{k}$ for any $k \in \mathbb{N}^{*}$,

4) $w_{i}\left(\partial / \partial w_{m}\right) \mathcal{G}_{k} \subset \mathcal{G}_{k}$ for any $k \in \mathbb{N}^{*}$ and $i, m \in \mathbb{N}$ with $0 \leq i \leq m$,

5) $(\partial / \partial x) \mathcal{G}_{k} \subset \mathcal{G}_{k}$ for any $k \in \mathbb{N}^{*}$, 
6) $\mathcal{G}_{k} \times \mathcal{G}_{l} \subset \mathcal{G}_{k+l}$ for any $k, l \in \mathbb{N}^{*}$,

7) $w_{m+1}\left(\partial / \partial w_{m}\right) \mathcal{G}_{k} \times \mathcal{G}_{l} \subset \mathcal{G}_{k+l}$ for any $m \in \mathbb{N}$ and $k, l \in \mathbb{N}^{*}$,

8) $w_{m+1}\left(\partial / \partial w_{m}\right) \mathcal{G}_{k} \times w_{p+1}\left(\partial / \partial w_{p}\right) \mathcal{G}_{l} \subset \mathcal{G}_{k+l}$ for any $m, p \in \mathbb{N}$ and $k, l \in \mathbb{N}^{*}$.

The conditions 7) and 8) imply that $D\left[\mathcal{G}_{k}\right] \times \mathcal{G}_{l} \subset \mathcal{G}_{k+l}$ and $D\left[\mathcal{G}_{k}\right] \times D\left[\mathcal{G}_{l}\right] \subset \mathcal{G}_{k+l}$ hold for any $k, l \in \mathbb{N}^{*}$. By combining these conditions with the construction of the formal solution we can obtain the result in Proposition 3.2.3.

\section{§3.3. Convergence of the formal solution of $(\Psi)$}

For $s>0$ and a function $\varphi(x) \in \mathcal{O}_{R}$ we define the norm $\|\varphi\|_{s}$ of $\varphi(x)$ by

$$
\|\varphi\|_{s}=\max _{|x| \leq s}|\varphi(x)| .
$$

For $c>0,0<s \leq R$ and

$$
f_{k}=\sum_{i+|j|=k} f_{i, j}(x) t^{i} w_{0}{ }^{j_{0}} w_{1}{ }^{j_{1}} \cdots w_{k-1}{ }^{j_{k-1}} \in \mathcal{H}_{k, R}\left[t, w_{0}, \ldots, w_{k-1}\right]
$$

we define the norm $\left\|f_{k}\right\|_{c, s}$ of $f_{k}$ by

$$
\left\|f_{k}\right\|_{c, s}=\sum_{i+|j|=k}\left\|f_{i, j}\right\|_{s}\left(\frac{0 !}{c^{0}}\right)^{j_{0}}\left(\frac{1 !}{c^{1}}\right)^{j_{1}}\left(\frac{2 !}{c^{2}}\right)^{j_{2}} \cdots\left(\frac{(k-1) !}{c^{k-1}}\right)^{j_{k-1}} .
$$

It is easy to see that $\left\|f_{k} g_{l}\right\|_{c, s} \leq\left\|f_{k}\right\|_{c, s}\left\|g_{l}\right\|_{c, s}$ holds for any $f_{k} \in \mathcal{H}_{k, R}[t$, $\left.u_{0}, \ldots, u_{k-1}\right]$ and $g_{l} \in \mathcal{H}_{l, R}\left[t, u_{0}, \ldots, u_{l-1}\right]$. We denote by $\mathbb{C}\{z\}$ the ring of convergent power series in $z$ with complex coefficients.

Let

$$
\psi=\sum_{k \geq 1} \psi_{k}\left(t, x, w_{0}, \ldots, w_{k-1}\right) \in \sum_{k \geq 1} \mathcal{H}_{k, R}\left[t, w_{0}, \ldots, w_{k-1}\right]
$$

be the formal solution constructed in Proposition 3.2.1. In this section we will show that this formal solution is convergent in the following sense:

Theorem 3.3.1. $\quad$ Suppose the conditions $\left.\left.\mathrm{A}_{1}\right), \mathrm{A}_{2}\right), \mathrm{A}_{3}$ ) and that

$$
\begin{aligned}
& |i+\lambda(x)(j-1)| \geq \sigma_{0}(i+j) \quad \text { on } D_{R} \\
& \quad \text { for any }(i, j) \in \mathbb{N} \times \mathbb{N} \backslash\{(0,0),(0,1)\}
\end{aligned}
$$

for some $\sigma_{0}>0$. Then we can find a constant $c_{0}>0$ such that the formal solution (3.3.1) satisfies

$$
\sum_{k \geq 1}\left\|\psi_{k}\right\|_{c, s} z^{k} \in \mathbb{C}\{z\} \quad \text { for any } 0<c \leq c_{0} \text { and } 0<s<R .
$$


Hence, we may say that the formal solution $\psi\left(t, x, w_{0}, w_{1}, \ldots\right)$ is convergent if

$$
\sum_{k \geq 1}\left\|\psi_{k}\right\|_{c, s} z^{k} \in \mathbb{C}\{z\}
$$

holds for some $c>0$ and $s>0$.

The rest part of this section will be used to prove Theorem 3.3.1. First we note:

Lemma 3.3.2. $\quad$ Suppose that the condition (3.3.2) holds for some $\sigma_{0}>$ 0 . Then we can find a constant $c_{0}>0$ which satisfies the following: if $k \geq 2$, $0<c \leq c_{0}$ and $0<s \leq R$, the unique solution $\psi_{k} \in \mathcal{H}_{k, R}\left[t, w_{0}, \ldots, w_{k-1}\right]$ of the equation $\left(\tau_{k}^{*}-\lambda(x)\right) \psi_{k}=f_{k} \in \mathcal{H}_{k, R}\left[t, w_{0}, \ldots, w_{k-1}\right]$ satisfies

$$
\sigma k\left\|\psi_{k}\right\|_{c, s} \leq\left\|f_{k}\right\|_{c, s}
$$

where $\sigma=\sigma_{0} / 2$.

Proof. We recall that $\lambda(x)$ is a holomorphic function in a neighborhood of $D_{R}$ and so we have

$$
\left\|\lambda_{m, p}\right\|_{R}=\frac{m !}{p !(m-p) !}\left\|\left(\frac{\partial}{\partial x}\right)^{m-p} \lambda\right\|_{R} \leq \frac{m !}{p !} \frac{\Lambda}{\eta^{m-p}}
$$

for some $\Lambda>0$ and $\eta>0$ which are independent of $m$ and $p$. We set $c_{0}=$ $\sigma_{0} \eta /\left(2 \Lambda+\sigma_{0}\right)$; then we see that the condition $0<c \leq c_{0}$ implies $0<c<\eta$ and $\Lambda(c / \eta) /(1-(c / \eta))=\Lambda c /(\eta-c) \leq \sigma_{0} / 2$.

Let $\psi_{k} \in \mathcal{H}_{k, R}\left[t, w_{0}, \ldots, w_{k-1}\right]$ and $f_{k} \in \mathcal{H}_{k, R}\left[t, w_{0}, \ldots, w_{k-1}\right]$ satisfy the equation $\left(\tau_{k}^{*}-\lambda(x)\right) \psi_{k}=f_{k}$. Set

$$
\begin{aligned}
\psi_{k} & =\sum_{i+|j|=k} \psi_{i, j}(x) t^{i} w_{0}^{j_{0}} \cdots w_{k-1}{ }^{j_{k-1}}, \\
f_{k} & =\sum_{i+|j|=k} f_{i, j}(x) t^{i} w_{0}^{j_{0}} \cdots w_{k-1}{ }^{j_{k-1}} .
\end{aligned}
$$

Then the equation $\left(\tau_{k}^{*}-\lambda(x)\right) \psi_{k}=f_{k}$ is equivalent to

$$
\begin{aligned}
& \sum_{i+|j|=k}(i+\lambda(x)(|j|-1)) \psi_{i, j}(x) t^{i} w_{0}^{j_{0}} \cdots w_{k-1}^{j_{k-1}} \\
& =\sum_{i+|j|=k} f_{i, j}(x) t^{i} w_{0}^{j_{0}} \cdots w_{k-1}{ }^{j_{k-1}} \\
& -\sum_{i+|j|=k} \sum_{1 \leq m \leq k-1} \sum_{0 \leq p \leq m-1} \lambda_{m, p}(x) \psi_{i, j}(x) \times \\
& \times j_{m} t^{i} w_{0}^{j_{0}} \cdots w_{p}^{j_{p}+1} \cdots w_{m}^{j_{m}-1} \cdots w_{k-1}^{j_{k-1}} .
\end{aligned}
$$


Therefore, by using the condition (3.3.2) and (3.3.5) we have

$$
\begin{aligned}
\sigma_{0} k\left\|\psi_{k}\right\|_{c, s} & \\
\leq\left\|f_{k}\right\|_{c, s}+ & \sum_{i+|j|=k} \sum_{1 \leq m \leq k-1} \sum_{0 \leq p \leq m-1} \frac{m !}{p !} \frac{\Lambda}{\eta^{m-p}}\left\|\psi_{i, j}\right\|_{s} \times \\
& \times j_{m}\left(\frac{0 !}{c^{0}}\right)^{j_{0}} \cdots\left(\frac{p !}{c^{p}}\right)^{j_{p}+1} \cdots\left(\frac{m !}{c^{m}}\right)^{j_{m}-1} \cdots\left(\frac{(k-1) !}{c^{k-1}}\right)^{j_{k-1}} \\
=\left\|f_{k}\right\|_{c, s}+ & \sum_{i+|j|=k} \sum_{1 \leq m \leq k-1} \sum_{0 \leq p \leq m-1} \frac{\Lambda}{\eta^{m-p}} c^{m-p}\left\|\psi_{i, j}\right\|_{s} \times \\
& \times j_{m}\left(\frac{0 !}{c^{0}}\right)^{j_{0}} \ldots\left(\frac{p !}{c^{p}}\right)^{j_{p}} \cdots\left(\frac{m !}{c^{m}}\right)^{j_{m}} \cdots\left(\frac{(k-1) !}{c^{k-1}}\right)^{j_{k-1}} .
\end{aligned}
$$

Here, we note: if $0<c \leq c_{0}$ we have

$$
\sum_{0 \leq p \leq m-1} \frac{\Lambda}{\eta^{m-p}} c^{m-p} \leq \sum_{q \geq 1} \Lambda(c / \eta)^{q}=\Lambda(c / \eta) /(1-(c / \eta)) \leq \sigma_{0} / 2 ;
$$

by applying this to (3.3.6) and by using $|j| \leq k$ we obtain

$$
\begin{aligned}
& \sigma_{0} k\left\|\psi_{k}\right\|_{c, s} \\
& \left.\leq\left\|f_{k}\right\|_{c, s}+\frac{\sigma_{0}}{2} \sum_{i+|j|=k}\left\|\psi_{i, j}\right\|_{s} \sum_{1 \leq m \leq k-1} j_{m}\right]\left(\frac{0 !}{c^{0}}\right)^{j_{0}}\left(\frac{1 !}{c^{1}}\right)^{j_{1}} \cdots\left(\frac{(k-1) !}{c^{k-1}}\right)^{j_{k-1}} \\
& \leq\left\|f_{k}\right\|_{c, s}+\frac{\sigma_{0}}{2} k \sum_{i+|j|=k}\left\|\psi_{i, j}\right\|_{s}\left(\frac{0 !}{c^{0}}\right)^{j_{0}}\left(\frac{1 !}{c^{1}}\right)^{j_{1}} \cdots\left(\frac{(k-1) !}{c^{k-1}}\right)^{j_{k-1}} \\
& =\left\|f_{k}\right\|_{c, s}+\frac{\sigma_{0}}{2} k\left\|\psi_{k}\right\|_{c, s} .
\end{aligned}
$$

This leads us to the estimate (3.3.4).

The following result is a modification of Nagumo's lemma.

Lemma 3.3.3. Let $c>0$ be fixed. If $\psi_{k} \in \mathcal{H}_{k, R}\left[t, w_{0}, \ldots, w_{k-1}\right]$ (with $k \geq 2)$ has the form (3.2.7) and if

$$
\left\|\psi_{k}\right\|_{c, s} \leq \frac{C}{(R-s)^{a}} \quad \text { for any } 0<s<R
$$

for some $C>0$ and $a \geq 0$, then we have

$$
\left\|D\left[\psi_{k}\right]\right\|_{c, s} \leq \frac{(a+1) e C}{(R-s)^{a+1}}+\frac{3 k}{c} \frac{C}{(R-s)^{a}} \quad \text { for any } 0<s<R .
$$


Proof. Set

$$
\psi_{k}=\sum_{(i, j) \in S_{k}} \psi_{i, j}(x) t^{i} w_{0}{ }^{j_{0}} w_{1}^{j_{1}} \cdots w_{k-1}{ }^{j_{k-1}}
$$

we have

$$
\begin{aligned}
D\left[\psi_{k}\right]= & \sum_{(i, j) \in S_{k}} \frac{\partial \psi_{i, j}}{\partial x}(x) t^{i} w_{0}{ }^{j_{0}} w_{1}^{j_{1}} \cdots w_{k-1} j_{k-1} \\
+ & \sum_{\substack{(i, j) \in S_{k} \\
|j| \geq 1}}\left[\sum_{0 \leq m \leq k-2} \psi_{i, j}(x) j_{m} t^{i} w_{0}{ }^{j_{0}} \cdots w_{m}{ }^{j_{m}-1} w_{m+1}{ }^{j_{m+1}+1} \cdots w_{k-1} j_{k-1}\right. \\
& \left.\quad+\psi_{i, j}(x) j_{k-1} t^{i} w_{0}^{j_{0}} w_{1}^{j_{1}} \cdots w_{k-2}{ }^{j_{k-2}} w_{k-1}{ }^{j_{k-1}-1} w_{k}\right]
\end{aligned}
$$

Therefore, for any $0<s<R$ and any $0<h<R-s$ we have

$$
\begin{aligned}
& \left\|D\left[\psi_{k}\right]\right\|_{c, s} \\
& \leq \sum_{(i, j) \in S_{k}}\left\|\frac{\partial \psi_{i, j}}{\partial x}\right\|_{s}\left(\frac{0 !}{c^{0}}\right)^{j_{0}}\left(\frac{1 !}{c^{1}}\right)^{j_{1}} \cdots\left(\frac{(k-1) !}{c^{k-1}}\right)^{j_{k-1}} \\
& +\sum_{\substack{(i, j) \in S_{k} \\
|j| \geq 1}}\left[\sum_{0 \leq m \leq k-2}\left\|\psi_{i, j}\right\|_{s} j_{m}\left(\frac{0 !}{c^{0}}\right)^{j_{0}} \cdots\left(\frac{m !}{c^{m}}\right)^{j_{m}-1} \times\right. \\
& \times\left(\frac{(m+1) !}{c^{m+1}}\right)^{j_{m+1}+1} \cdots\left(\frac{(k-1) !}{c^{k-1}}\right)^{j_{k-1}} \\
& \left.+\left\|\psi_{i, j}\right\|_{s} j_{k-1}\left(\frac{0 !}{c^{0}}\right)^{j_{0}} \cdots\left(\frac{(k-2) !}{c^{k-2}}\right)^{j_{k-2}}\left(\frac{(k-1) !}{c^{k-1}}\right)^{j_{k-1}-1}\left(\frac{k !}{c^{k}}\right)\right] \\
& \leq \sum_{(i, j) \in S_{k}} \frac{1}{h}\left\|\psi_{i, j}\right\|_{s+h}\left(\frac{0 !}{c^{0}}\right)^{j_{0}}\left(\frac{1 !}{c^{1}}\right)^{j_{1}} \cdots\left(\frac{(k-1) !}{c^{k-1}}\right)^{j_{k-1}} \\
& +\sum_{\substack{(i, j) \in S_{k} \\
|j| \geq 1}} \sum_{0 \leq m \leq k-1}\left\|\psi_{i, j}\right\|_{s} j_{m} \frac{(m+1)}{c}\left(\frac{0 !}{c^{0}}\right)^{j_{0}} \cdots\left(\frac{m !}{c^{m}}\right)^{j_{m}} \times \\
& \times\left(\frac{(m+1) !}{c^{m+1}}\right)^{j_{m+1}} \cdots\left(\frac{(k-1) !}{c^{k-1}}\right)^{j_{k-1}} .
\end{aligned}
$$

Since $(i, j) \in S_{k}$ and $|j| \geq 1$ imply $i+2(|j|-1) \geq\langle j\rangle$, we have

$$
\sum_{0 \leq m \leq k-1} j_{m}(m+1)=\langle j\rangle+|j| \leq i+2(|j|-1)+|j| \leq 3(i+|j|)=3 k
$$


and so by (3.3.7) and (3.3.9) we see

$$
\begin{aligned}
\left\|D\left[\psi_{k}\right]\right\|_{c, s} \leq & \sum_{(i, j) \in S_{k}} \frac{1}{h}\left\|\psi_{i, j}\right\|_{s+h}\left(\frac{0 !}{c^{0}}\right)^{j_{0}}\left(\frac{1 !}{c^{1}}\right)^{j_{1}} \cdots\left(\frac{(k-1) !}{c^{k-1}}\right)^{j_{k-1}} \\
& +\frac{3 k}{c} \sum_{\substack{(i, j) \in S_{k} \\
|j| \geq 1}}\left\|\psi_{i, j}\right\|_{s}\left(\frac{0 !}{c^{0}}\right)^{j_{0}}\left(\frac{1 !}{c^{1}}\right)^{j_{1}} \cdots\left(\frac{(k-1) !}{c^{k-1}}\right)^{j_{k-1}} \\
\leq & \frac{1}{h}\left\|\psi_{k}\right\|_{c, s+h}+\frac{3 k}{c}\left\|\psi_{k}\right\|_{c, s} \\
\leq & \frac{1}{h} \frac{C}{(R-s-h)^{a}}+\frac{3 k}{c} \frac{C}{(R-s)^{a}} .
\end{aligned}
$$

In the case $a=0$, by letting $h \longrightarrow R-s$ we have the result

$$
\left\|D\left[\psi_{k}\right]\right\|_{c, s} \leq \frac{C}{(R-s)}+\frac{3 k}{c} C,
$$

which proves (3.3.8) with $a=0$. In the case $a>0$, by taking $h=(R-s) /(a+1)$ we obtain

$$
\begin{aligned}
\left\|D\left[\psi_{k}\right]\right\|_{c, s} & \leq \frac{(a+1) C}{(R-s)^{a+1}} \frac{1}{(1-1 /(a+1))^{a}}+\frac{3 k}{c} \frac{C}{(R-s)^{a}} \\
& =\frac{(a+1) C}{(R-s)^{a+1}}\left(1+\frac{1}{a}\right)^{a}+\frac{3 k}{c} \frac{C}{(R-s)^{a}} \\
& \leq \frac{(a+1) e C}{(R-s)^{a+1}}+\frac{3 k}{c} \frac{C}{(R-s)^{a}},
\end{aligned}
$$

which proves (3.3.8).

Now, let us prove Theorem 3.3.1. Recall that our coupling equation $(\Psi)$ is written in the form

$$
\left(\tau^{*}-\lambda(x)\right) \psi=a(x) t+\sum_{i+j+\alpha \geq 2} c_{i, j, \alpha}(x) t^{i} \psi^{j}(D[\psi])^{\alpha} .
$$

We set $\left\|c_{i, j, \alpha}\right\|_{R}=A_{i, j, \alpha}($ for $i+j+\alpha \geq 2)$; then we see that the series

$$
\sum_{i+j+\alpha \geq 2} A_{i, j, \alpha} t^{i} Y^{j+\alpha}
$$

is convergent in a neighborhood of $(t, Y)=(0,0) \in \mathbb{C} \times \mathbb{C}$. For simplicity we may suppose that $0<R \leq 1$ holds.

Let

$$
\psi=\sum_{k \geq 1} \psi_{k}\left(t, x, w_{0}, \ldots, w_{k-1}\right) \in \sum_{k \geq 1} \mathcal{H}_{k, R}\left[t, w_{0}, \ldots, w_{k-1}\right]
$$


be the formal solution constructed in Proposition 3.2.1. We know that $\psi_{k}$ (for $k \geq 2$ ) are determined by the recurrent formulas:

$$
\begin{array}{r}
\left(\tau_{k}^{*}-\lambda(x)\right) \psi_{k}=\sum_{2 \leq i+j+\alpha \leq k} c_{i, j, \alpha}(x) t^{i}\left[\sum_{|p(j)|+|q(\alpha)|=k-i} \psi_{p_{1}} \times\right. \\
\left.\times \cdots \times \psi_{p_{j}} \times D\left[\psi_{q_{1}}\right] \times \cdots D\left[\psi_{q_{\alpha}}\right]\right] .
\end{array}
$$

Let $c_{0}>0$ be the constant in Lemma 3.3.2, and let $0<c \leq c_{0}$ be fixed. Take a positive constant $A>0$ so that

$$
\left\|\psi_{1}\right\|_{c, R} \leq A \quad \text { and } \quad\left\|D\left[\psi_{1}\right]\right\|_{c, R} \leq(e+3 / c) A
$$

hold. As a majorant equation of the formal solution (3.3.10), we consider the following functional equation with respect to $Y$ :

$$
Y=A z+\frac{1}{\sigma} \frac{1}{(R-s)} \sum_{i+j+\alpha \geq 2} \frac{A_{i, j, \alpha}}{(R-s)^{i+j+\alpha-2}} z^{i} Y^{j}((e+3 / c) Y)^{\alpha}
$$

where $\sigma=\sigma_{o} / 2$, and $s$ is a parameter with $0<s<R$. It is easy to see that (3.3.13) is an analytic functional equation with respect to $(z, Y)$. The implicit function theorem tells us that for any $0<s<R$ this equation (3.3.13) has a unique holomorphic solution $Y=Y(z)$ of the form

$$
Y=\sum_{k \geq 1} Y_{k} z^{k}
$$

Moreover, $Y_{k}(k=1,2, \ldots)$ are determined by the following recurrent formulas:

$$
Y_{1}=A
$$

and for $k \geq 2$

$$
\begin{aligned}
Y_{k}=\frac{1}{\sigma} \frac{1}{(R-s)} & \sum_{2 \leq i+j+\alpha \leq k} \frac{A_{i, j, \alpha}}{(R-s)^{i+j+\alpha-2}}\left[\sum_{|p(j)|+|q(\alpha)|=k-i} Y_{p_{1}} \times\right. \\
& \left.\times \cdots \times Y_{p_{j}} \times\left((e+3 / c) Y_{q_{1}}\right) \cdots\left((e+3 / c) Y_{q_{\alpha}}\right)\right] .
\end{aligned}
$$

By induction we can see also that $Y_{k}$ has the form

$$
Y_{k}=\frac{C_{k}}{(R-s)^{k-1}}, \quad k=1,2, \ldots
$$


for some constants $C_{1}=A$ and $C_{k} \geq 0(k \geq 2)$ which are independent of the parameter $s$. We often write $Y_{k}=Y_{k}(s)$; this emphasizes that $Y_{k}$ depends on the parameter $s$.

The following lemma guarantees that $Y(z)$ is a majorant series of the formal solution (3.3.10).

Lemma 3.3.4. Let $\psi=\sum_{k \geq 1} \psi_{k}$ be the formal solution (3.3.10). Then, for any $k=1,2, \ldots$ we have

$$
\begin{aligned}
\left\|\psi_{k}\right\|_{c, s} & \leq Y_{k}(s) \quad \text { for any } 0<s<R \\
\left\|D\left[\psi_{k}\right]\right\|_{c, s} & \leq(e+3 / c) Y_{k}(s) \quad \text { for any } 0<s<R .
\end{aligned}
$$

Proof. Since $Y_{1}=A$, the case $k=1$ is clear from (3.3.12). The general case is proved by induction on $k$.

Let $k \geq 2$ and suppose that $(3.3 .16)_{l}$ and $(3.3 .17)_{l}$ are already proved for $l=1,2, \ldots, k-1$. Then, by applying Lemma 3.3.2 to (3.3.11) and by using the induction hypothesis we have

$$
\begin{aligned}
\left\|\psi_{k}\right\|_{c, s} \leq \frac{1}{\sigma k} \sum_{2 \leq i+j+\alpha \leq k} A_{i, j, \alpha}\left[\sum_{|p(j)|+|q(\alpha)|=k-i}\left\|\psi_{p_{1}}\right\|_{c, s} \times\right. \\
\left.\quad \times \cdots \times\left\|\psi_{p_{j}}\right\|_{c, s} \times\left\|D\left[\psi_{q_{1}}\right]\right\|_{c, s} \cdots\left\|D\left[\psi_{q_{\alpha}}\right]\right\|_{c, s}\right] \\
\leq \frac{1}{\sigma k} \sum_{2 \leq i+j+\alpha \leq k} A_{i, j, \alpha}\left[\sum_{|p(j)|+|q(\alpha)|=k-i} Y_{p_{1}}(s) \times\right. \\
\left.\times \cdots \times Y_{p_{j}}(s) \times(e+3 / c) Y_{q_{1}}(s) \cdots(e+3 / c) Y_{q_{\alpha}}(s)\right] .
\end{aligned}
$$

Hence, comparing this with (3.3.14) we obtain

$$
\left\|\psi_{k}\right\|_{c, s} \leq \frac{(R-s)}{k} Y_{k}(s)=\frac{1}{k} \frac{C_{k}}{(R-s)^{k-2}}
$$

for any $0<s<R$. Since $1 / k \leq 1$ and $1 /(R-s) \geq 1$ hold, by (3.3.18) we have

$$
\left\|\psi_{k}\right\|_{c, s} \leq \frac{C_{k}}{(R-s)^{k-1}}=Y_{k}(s) \text { for any } 0<s<R
$$


which proves $(3.3 .16)_{k}$. Moreover, by applying Lemma 3.3.3 to (3.3.18) we have

$$
\begin{aligned}
\left\|D\left[\psi_{k}\right]\right\|_{c, s} & \leq \frac{(k-1) e}{k} \frac{C_{k}}{(R-s)^{k-1}}+\frac{3 k}{c k} \frac{C_{k}}{(R-s)^{k-2}} \\
& \leq e \frac{C_{k}}{(R-s)^{k-1}}+\frac{3}{c} \frac{C_{k}}{(R-s)^{k-1}} \\
& =(e+3 / c) Y_{k}(s) \quad \text { for any } 0<s<R
\end{aligned}
$$

which proves $(3.3 .17)_{k}$.

Thus, by induction on $k$ we have proved Lemma 3.3.4.

Let us return to the proof of Theorem 3.3.1. By Lemma 3.3.4 we have

$$
\sum_{k \geq 1}\left\|\psi_{k}\right\|_{c, s} z^{k} \ll \sum_{k \geq 1} Y_{k}(s) z^{k} .
$$

Since the right-hand side is convergent in a neighborhood of $z=0$, this completes the proof of Theorem 3.3.1.

Let us give a variant. Let

$$
\tau^{0}=t \frac{\partial}{\partial t}+\sum_{m \geq 0} w_{m} \frac{\partial}{\partial w_{m}}
$$

and let $G\left(t, x, u_{0}, u_{1}\right)$ be a holomorphic function defined in a neighborhood of the origin $\left(t, x, u_{0}, u_{1}\right)=(0,0,0,0) \in \mathbb{C}^{4}$ whose Taylor expansion in $\left(t, u_{0}, u_{1}\right)$ has the form

$$
G\left(t, x, u_{0}, u_{1}\right)=\sum_{i+j+\alpha \geq 2} \frac{A_{i, j, \alpha}}{(R-x)} t^{i} u_{0}{ }^{j} u_{1}^{\alpha}
$$

with $A_{i, j, \alpha} \geq 0(i+j+\alpha \geq 2)$ and $0<R \leq 1$. Let us consider the following equation

$$
\left(\tau^{0}-1\right) \psi=G(t, x, \psi, D[\psi])
$$

For $R>0$ we write

$$
\mathcal{H}_{k,(R)}\left[t, w_{0}, \ldots, w_{k-1}\right]=\bigcap_{0<\eta<R} \mathcal{H}_{k, \eta}\left[t, w_{0}, \ldots, w_{k-1}\right]
$$

We have 
Theorem 3.3.5. The equation (3.3.21) has a unique formal solution of the form $\psi=w_{0}+\sum_{k \geq 2} \psi_{k}\left(t, x, w_{0}, \ldots, w_{k-1}\right) \in \sum_{k \geq 1} \mathcal{H}_{k,(R)}\left[t, w_{0}, \ldots, w_{k-1}\right]$. Moreover we have

$$
\sum_{k \geq 1}\left\|\psi_{k}\right\|_{c, s} z^{k} \in \mathbb{C}\{z\} \quad \text { for any } c>0 \text { and } 0<s<R
$$

where $\psi_{1}=w_{0}$.

Proof. Set

$$
\psi=\sum_{k \geq 1} \psi_{k}\left(t, x, w_{0}, \ldots, w_{k-1}\right) \in \sum_{k \geq 1} \mathcal{H}_{k,(R)}\left[t, w_{0}, \ldots, w_{k-1}\right] .
$$

It is easy to see that under the condition $\psi_{1}=w_{0}$ the equation (3.3.21) has a unique formal solution of the form $(3.3 .22)$, and that $\psi_{k}\left(t, x, w_{0}, \ldots, w_{k-1}\right)$ (for $k \geq 2$ ) are determined by the following recurrent formulas:

$$
\psi_{k}=\frac{1}{k-1} \sum_{2 \leq i+j+\alpha \leq k} \frac{A_{i, j, \alpha}}{(R-x)} t^{i} \sum_{|p(j)|+|q(\alpha)|=k-i} \psi_{p_{1}} \cdots \psi_{p_{j}} \times D\left[\psi_{q_{1}}\right] \cdots D\left[\psi_{q_{\alpha}}\right] .
$$

Therefore, for any $c>0$ and any $0<s<R$ we have

$$
\begin{aligned}
\left\|\psi_{k}\right\|_{c, s} \leq \frac{1}{k-1} & \sum_{2 \leq i+j+\alpha \leq k} \frac{A_{i, j, \alpha}}{(R-s)}\left[\sum_{|p(j)|+|q(\alpha)|=k-i}\left\|\psi_{p_{1}}\right\|_{c, s} \times\right. \\
& \left.\times \cdots \times\left\|\psi_{p_{j}}\right\|_{c, s} \times\left\|D\left[\psi_{q_{1}}\right]\right\|_{c, s} \cdots\left\|D\left[\psi_{q_{\alpha}}\right]\right\|_{c, s}\right] .
\end{aligned}
$$

Let us consider now the functional equation with respect to $Y$ :

$$
Y=z+\frac{1}{(R-s)} \sum_{i+j+\alpha \geq 2} \frac{A_{i, j, \alpha}}{(R-s)^{1+2(i+j+\alpha-2)}} z^{i} Y^{j}((2 e+6 / c) Y)^{\alpha}
$$

where $s$ is a parameter with $0<s<R$. It is easy to see that (3.3.24) is an analytic functional equation with respect to $(z, Y)$. The implicit function theorem tells us that for any $0<s<R$ this equation (3.3.24) has a unique holomorphic solution $Y=Y(z)$ of the form

$$
Y=\sum_{k \geq 1} Y_{k} z^{k}
$$

Moreover, $Y_{k}(k=1,2, \ldots)$ are determined by the following recurrent formulas:

$$
Y_{1}=1
$$


and for $k \geq 2$

$$
\begin{gathered}
Y_{k}=\frac{1}{(R-s)} \sum_{2 \leq i+j+\alpha \leq k} \frac{A_{i, j, \alpha}}{(R-s)^{1+2(i+j+\alpha-2)}}\left[\sum_{|p(j)|+|q(\alpha)|=k-i} Y_{p_{1}} \times\right. \\
\left.\times \cdots \times Y_{p_{j}} \times\left((2 e+6 / c) Y_{q_{1}}\right) \cdots\left((2 e+6 / c) Y_{q_{\alpha}}\right)\right] .
\end{gathered}
$$

By induction we can see also that $Y_{k}$ has the form

$$
Y_{k}=\frac{C_{k}}{(R-s)^{2(k-1)}}, \quad k=1,2, \ldots
$$

for some constants $C_{1}=1$ and $C_{k} \geq 0(k \geq 2)$ which are independent of the parameter $s$. We write $Y_{k}=Y_{k}(s)$ when we emphasize the fact that $Y_{k}$ depends on the parameter $s$.

Since $\psi_{1}=w_{0}$ we have $D\left[\psi_{1}\right]=w_{1}$; therefore $\left\|\psi_{1}\right\|_{c, s}=1=Y_{1}$ and $\left\|D\left[\psi_{1}\right]\right\|_{c, s}=1 / c \leq(2 e+6 / c)=(2 e+6 / c) Y_{1}$ hold for any $0<s<R$. Moreover, for $k=2,3, \ldots$ we can prove the estimates

$$
\begin{aligned}
\left\|\psi_{k}\right\|_{c, s} & \leq Y_{k}(s) \quad \text { for any } 0<s<R \\
\left\|D\left[\psi_{k}\right]\right\|_{c, s} & \leq(2 e+6 / c) Y_{k}(s) \quad \text { for any } 0<s<R
\end{aligned}
$$

by induction on $k$ in the same way as in the proof of Lemma 3.3.4. The factor $(2 e+6 / c)$ comes from the estimate $((2 k-2) e+3 k / c) /(k-1)=2 e+(k /(k-$ 1)) $3 / c \leq 2 e+6 / c$ for any $k \geq 2$.

Thus, we have

$$
\sum_{k \geq 1}\left\|\psi_{k}\right\|_{c, s} z^{k} \ll \sum_{k \geq 1} Y_{k}(s) z^{k}
$$

Since the right-hand side is convergent in a neighborhood of $z=0$, this completes the proof of Theorem 3.3.5.

\section{§3.4. Convergence of the formal solution of $(\Phi)$}

For $r>0, c>0,0<s \leq R$ and

$$
f_{k}=\sum_{i+|j|=k} f_{i, j}(x) t^{i} u_{0}{ }^{j_{0}} u_{1}{ }^{j_{1}} \cdots u_{k-1}{ }^{j_{k-1}} \in \mathcal{H}_{k, R}\left[t, u_{0}, \ldots, u_{k-1}\right]
$$

we define the norm $\left\|f_{k}\right\|_{r, c, s}$ of $f_{k}$ by

$$
\left\|f_{k}\right\|_{r, c, s}=\sum_{i+|j|=k}\left\|f_{i, j}\right\|_{s} r^{i}\left(\frac{0 !}{c^{0}}\right)^{j_{0}}\left(\frac{1 !}{c^{1}}\right)^{j_{1}}\left(\frac{2 !}{c^{2}}\right)^{j_{2}} \cdots\left(\frac{(k-1) !}{c^{k-1}}\right)^{j_{k-1}} .
$$


In Proposition 3.1.1 we have shown that under the assumption (3.1.1) the coupling equation $(\Phi)$ has a family of formal solutions of the form

$$
\phi=-\alpha(x) \beta(x) t+\beta(x) u_{0}+\sum_{k \geq 2} \phi_{k}\left(t, x, u_{0}, \ldots, u_{k-1}\right)
$$

with $\alpha(x)=a(x) /(1-\lambda(x)), \beta(x) \in \mathcal{O}_{R}$ is arbitrary, and $\phi_{k}\left(t, x, u_{0}, \ldots, u_{k-1}\right)$ $\in \mathcal{H}_{k, R}\left[t, u_{0}, \ldots, u_{k-1}\right](k=2,3, \ldots)$ : we know that the terms $\phi_{k}(k \geq 2)$ are determined by the recurrent formulas

$$
\begin{aligned}
& \left(\tau_{k}-\lambda(x)\right) \phi_{k} \\
& =-\sum_{1 \leq q \leq k-1} \sum_{0 \leq m \leq q-1} D^{m}\left[R_{k-q+1}\right]\left(t, x, u_{0}, \ldots, u_{m+1}\right) \frac{\partial \phi_{q}}{\partial u_{m}}
\end{aligned}
$$

where

$$
\tau_{k}=t \frac{\partial}{\partial t}+\sum_{0 \leq m \leq k-1}\left(a^{(m)}(x) t+\sum_{0 \leq i \leq m} \lambda_{m, i}(x) u_{i}\right) \frac{\partial}{\partial u_{m}}, \quad k \geq 2 .
$$

In this section we will prove the convergence of this formal solution (3.4.1) in the following sense:

Theorem 3.4.1. Suppose the conditions $\left.\left.\left.\mathrm{A}_{1}\right), \mathrm{A}_{2}\right), \mathrm{A}_{3}\right)$ and that

$$
\begin{aligned}
& |i+\lambda(x)(j-1)| \geq \sigma_{0}(i+j) \quad \text { on } D_{R} \\
& \quad \text { for any }(i, j) \in \mathbb{N} \times \mathbb{N} \backslash\{(0,0),(0,1)\}
\end{aligned}
$$

for some $\sigma_{0}>0$. Then we can find constants $r_{0}>0$ and $0<c_{0}<R$ such that the formal solution (3.4.1) satisfies

$$
\sum_{k \geq 1}\left\|\phi_{k}\right\|_{r, c, s} z^{k} \in \mathbb{C}\{z\}
$$

$\left(\right.$ with $\left.\phi_{1}=-\alpha(x) \beta(x) t+\beta(x) u_{0}\right)$ for any $0<r \leq r_{0}, 0<c \leq c_{0}$ and $0<s<R$ satisfying $c+s=R$.

The rest part of this section is used to prove this result. In the proof, we may suppose:

$$
\begin{aligned}
\left\|a^{(m)}\right\|_{R} & \leq \frac{m ! A}{\eta^{m}}, \quad m=0,1,2, \ldots \\
\left\|\lambda_{m, p}\right\|_{R} & \leq \frac{m !}{p !} \frac{\Lambda}{\eta^{m-p}}, \quad m=0,1,2, \ldots \text { and } 0 \leq p \leq m
\end{aligned}
$$


for some $A>0, \eta>0$ and $\Lambda>0$ which are independent of $m$ and $p$. As is proved later, it is sufficient to take $r_{0}>0$ and $0<c_{0}<R$ so that

$$
0<c_{0}<\eta \text { and } r_{0} A+\left(\Lambda c_{0} /\left(\eta-c_{0}\right)\right) \leq \sigma_{0} / 2
$$

hold: we fix such $r_{0}>0$ and $0<c_{0}<R$ from now.

\subsubsection{A reduction}

First, let us recall that $R_{p}\left(t, x, u_{0}, u_{1}\right)$ in the right-hand side of (3.4.2) is expressed in the form

$$
R_{p}\left(t, x, u_{0}, u_{1}\right)=\sum_{i+j+\alpha=p} c_{i, j, \alpha}(x) t^{i} u_{0}^{j} u_{1}^{\alpha}, \quad p \geq 2,
$$

where $c_{i, j, \alpha}(x)(i+j+\alpha \geq 2)$ are holomorphic functions in a neighborhood of $D_{R}$. Without loss of generality we may suppose: $0<R \leq 1$. We set $\left\|c_{i, j, \alpha}\right\|_{R}=A_{i, j, \alpha}$; then we have $c_{i, j, \alpha}(x) \ll A_{i, j, \alpha} /(1-x / R) \ll A_{i, j, \alpha} /(R-x)$. We set

$$
G\left(t, x, w_{0}, w_{1}\right)=\sum_{i+j+\alpha \geq 2} \frac{A_{i, j, \alpha}}{(R-x)} t^{i} w_{0}^{j} w_{1}^{\alpha}:
$$

we have $R_{p}\left(t, x, w_{0}, w_{1}\right) \ll G\left(t, x, w_{0}, w_{1}\right)$ for all $p \geq 2$ as power series with respect to $\left(t, x, w_{0}, w_{1}\right)$. As in section 3.3 we use the notation

$$
\mathcal{H}_{k,(R)}\left[t, w_{0}, \ldots, w_{k-1}\right]=\bigcap_{0<\eta<R} \mathcal{H}_{k, \eta}\left[t, w_{0}, \ldots, w_{k-1}\right] .
$$

Under these notations, let us consider the following equation

$$
\left(\tau^{0}-1\right) \psi^{+}=G\left(t, x, \psi^{+}, D\left[\psi^{+}\right]\right),
$$

where $\tau^{0}$ is the vector field in (3.3.19), that is,

$$
\tau^{0}=t \frac{\partial}{\partial t}+\sum_{m \geq 0} w_{m} \frac{\partial}{\partial w_{m}} .
$$

It is easy to see that this equation (3.4.6) has a family of formal solutions of the form

$$
\psi^{+}=\sum_{k \geq 1} \psi_{k}^{+}\left(t, x, w_{0}, \ldots, w_{k-1}\right) \in \sum_{k \geq 1} \mathcal{H}_{k,(R)}\left[t, w_{0}, \ldots, w_{k-1}\right]:
$$


moreover we see that $\psi_{1}^{+} \in \mathcal{H}_{1,(R)}\left[t, w_{0}\right]$ can be chosen arbitrarily and the other terms $\psi_{k}^{+}(k \geq 2)$ are determined by the recurrent formulas:

$$
\begin{aligned}
\psi_{k}^{+}=\frac{1}{k-1} \sum_{2 \leq i+j+\alpha \leq k} \frac{A_{i, j, \alpha}}{(R-x)} t^{i}\left[\sum_{|p(j)|+|q(\alpha)|=k-i} \psi_{p_{1}}^{+} \times\right. \\
\times \cdots \times \psi_{p_{j}}^{+} \times D\left[\psi_{q_{1}}^{+}\right] \times \cdots \times D\left[\psi_{q_{\alpha}}^{+}\right] .
\end{aligned}
$$

We take $\psi_{1}^{+}=w_{0}$ from now. Then, on the convergence of this formal solution (3.4.7) (with $\psi_{1}^{+}=w_{0}$ ), by Theorem 3.3.5 we have

Proposition 3.4.2. For any $r>0, c>0$ and $0<s<R$ we have

$$
\sum_{k \geq 1}\left\|\psi_{k}^{+}\right\|_{r, c, s} z^{k} \in \mathbb{C}\{z\} .
$$

By combining this with the Stirling's formula we can see also

$$
\sum_{k \geq 1} \frac{(k-1)^{k-1}}{(k-1) !}\left\|\psi_{k}^{+}\right\|_{r, c, s} z^{k} \in \mathbb{C}\{z\}
$$

where in the case $k=1$ the notation $(k-1)^{k-1}$ should be read as $0^{0}=1$. Thus, to prove Theorem 3.4.1 it is sufficient to show the following inequality (3.4.9) and (3.4.10):

Proposition 3.4.3. Let $\phi=\sum_{k \geq 1} \phi_{k}$ be the formal solution (3.4.1) with $\phi_{1}=-\alpha(x) \beta(x) t+\beta(x) u_{0}$, and let $\psi^{+}=\sum_{k \geq 1} \psi_{k}^{+}$be the formal solution of (3.4.6) with $\psi_{1}^{+}=w_{0}$. Then, we have

$$
\begin{aligned}
\left\|\phi_{1}\right\|_{r, c, s} & \leq\|\beta\|_{s}\left(\|\alpha\|_{s} r+\left\|\psi_{1}^{+}\right\|_{r, c, s}\right), \\
\left\|\phi_{k}\right\|_{r, c, s} & \leq \frac{(k-1)^{k-1}}{\sigma^{k-1}(k-1) !}\|\beta\|_{s}\left\|\psi_{k}^{+}\right\|_{r, c, s} \quad \text { for } k \geq 2
\end{aligned}
$$

for any $0<r \leq r_{0}, 0<c \leq c_{0}, 0<s<R$ satisfying $c+s=R$, and $0<\sigma \leq \min \left\{1, \sigma_{0} / 2\right\}$.

\subsubsection{Basic properties of $\psi^{+}$}

Let $\psi^{+}=\sum_{p \geq 1} \psi_{p}^{+}\left(t, x, w_{0}, \ldots, w_{p-1}\right)$ be the formal solution of (3.4.6) with $\psi_{1}^{+}=w_{0}$ and $\psi_{p}^{+}\left(t, x, w_{0}, \ldots, w_{p-1}\right) \in \mathcal{H}_{p,(R)}\left[t, w_{0}, \ldots, w_{p-1}\right]($ for $p \geq 2)$. We have 
Lemma 3.4.4. (1) $\psi_{k}^{+}\left(t, x, w_{0}, \ldots, w_{k-1}\right)($ for $k \geq 2)$ has the form

$$
\psi_{k}^{+}=\sum_{i+|j|=k} \sum_{1 \leq l \leq 2 k-3} \frac{C_{i, j, l}}{(R-x)^{l}} t^{i} w_{0}^{j_{0}} \cdots w_{k-1} j_{k-1} \gg 0
$$

with $C_{i, j, l} \geq 0(i+|j|=k$ and $1 \leq l \leq 2 k-3)$.

(2) If $r>0, c>0$ and $s>0$ hold, we have

$$
\rho \ll_{\rho} \frac{c^{m}}{m !} D^{m}\left[\psi^{+}\right]\left(r \rho, s, \frac{0 !}{c^{0}} \rho, \frac{1 !}{c^{1}} \rho, \frac{2 !}{c^{2}} \rho, \ldots\right)
$$

for any $m=0,1,2, \ldots$, where $\ll_{\rho}$ denotes the majorant relation in $\mathbb{C}[[\rho]]$ the ring of formal power series in $\rho$.

(3) If $r>0,0<c<R, 0<s<R$ and $c+s=R$ hold, we have

$$
\begin{aligned}
D^{i}\left[\psi^{+}\right] & \left(r \rho, s, \frac{0 !}{c^{0}} \rho, \frac{1 !}{c^{1}} \rho, \frac{2 !}{c^{2}} \rho, \ldots\right) \\
& \ll \rho \frac{i !}{m !} c^{m-i} D^{m}\left[\psi^{+}\right]\left(r \rho, s, \frac{0 !}{c^{0}} \rho, \frac{1 !}{c^{1}} \rho, \frac{2 !}{c^{2}} \rho, \ldots\right)
\end{aligned}
$$

for any $0 \leq i \leq m$.

Proof. The part (1) is verified by induction on $k$; in the argument we should use (3.4.8) and the following facts $\psi_{1}^{+}=w_{0}, D\left[\psi_{1}^{+}\right]=w_{1}, 2 k-3 \leq$ $2(k-1),(2 k-3)+1=2(k-1)$, and

$$
\begin{aligned}
& 1+\sum_{1 \leq m \leq j} 2\left(p_{m}-1\right)+\sum_{1 \leq n \leq \alpha} 2\left(q_{n}-1\right) \\
& =1+2|p(j)|-2 j+2|q(\alpha)|-2 \alpha \\
& =1+2(i+|p(j)|+|q(\alpha)|)-2 i-2 j-2 \alpha=1+2 k-2 i-2 j-2 \alpha \\
& =(2 k-3)-2(i+j+\alpha-2) \leq 2 k-3 .
\end{aligned}
$$

Since $\psi_{1}^{+}=w_{1}$, we have $D^{m}\left[\psi_{1}^{+}\right]=w_{m}$ and so

$$
\begin{aligned}
D^{m}\left[\psi^{+}\right] & \left(r \rho, s, \frac{0 !}{c^{0}} \rho, \frac{1 !}{c^{1}} \rho, \frac{2 !}{c^{2}} \rho, \ldots\right) \\
& \gg_{\rho} D^{m}\left[\psi_{1}^{+}\right]\left(r \rho, s, \frac{0 !}{c^{0}} \rho, \frac{1 !}{c^{1}} \rho, \frac{2 !}{c^{2}} \rho, \ldots\right)=\frac{m !}{c^{m}} \rho ;
\end{aligned}
$$

this proves the part (2).

Let us show (3). We note that to verify the condition (3.4.12) it is enough to discuss only the following two cases:

Case (i) $\psi^{+}=w_{0}$, and 
Case (ii) $q+|j|=k \geq 2$ and $\psi^{+}$has the form

$$
\psi^{+}=\frac{C}{(R-x)^{l}} t^{q} w_{0}^{j_{0}} \cdots w_{k-1}^{j_{k-1}} \quad \text { with } C \geq 0 \text { and } l \geq 1 .
$$

Take any $r>0,0<c<R$ and $0<s<R$ with $c+s=R$; for simplicity we write

$$
\left(*_{\rho}\right)=\left(r \rho, s, \frac{0 !}{c^{0}} \rho, \frac{1 !}{c^{1}} \rho, \frac{2 !}{c^{2}} \rho, \ldots\right) .
$$

In the case $\psi^{+}=w_{0}$ we have $D^{i}\left[\psi^{+}\right]=w_{i}$ and $D^{m}\left[\psi^{+}\right]=w_{m}$; therefore if $0 \leq i \leq m$ we have (3.4.12) by

$$
D^{i}\left[\psi^{+}\right]\left(*_{\rho}\right)=\frac{i !}{c^{i}} \rho=\frac{i !}{m !} c^{m-i} \frac{m !}{c^{m}} \rho=\frac{i !}{m !} c^{m-i} D^{m}\left[\psi^{+}\right]\left(*_{\rho}\right) .
$$

Let us show the case (ii). Suppose that $q+|j|=k \geq 2$ and that $\psi^{+}$has the form (3.4.13). Set $u(x)=c /(R-x)$; then we have $u^{(n)}(x)=n ! c /(R-x)^{n+1}$ for $n=0,1,2, \ldots$, and therefore

$$
\begin{aligned}
\psi^{+} & \left(t, x, u(x), u^{(1)}(x), \ldots, u^{(k-1)}(x)\right) \\
& =\psi^{+}\left(t, x, \frac{0 ! c}{(R-x)}, \frac{1 ! c}{(R-x)^{2}}, \ldots, \frac{(k-1) ! c}{(R-x)^{k}}\right) \\
& =\frac{C}{(R-x)^{l}} t^{q}\left(\frac{0 ! c}{(R-x)}\right)^{j_{0}} \cdots\left(\frac{(k-1) ! c}{(R-x)^{k}}\right)^{j_{k-1}}=\frac{M}{(R-x)^{p}} t^{q}
\end{aligned}
$$

where $M=C(0 ! c)^{j_{0}} \cdots((k-1) ! c)^{j_{k-1}}$ and $p=l+j_{0}+2 j_{1}+\cdots+k j_{k-1} \geq 1$. By the definition of $D$ we have

$$
\begin{aligned}
D^{i} & {\left[\psi^{+}\right]\left(t, x, \frac{0 ! c}{(R-x)}, \frac{1 ! c}{(R-x)^{2}}, \ldots, \frac{(k-1+i) ! c}{(R-x)^{k+i}}\right) } \\
& =D^{i}\left[\psi^{+}\right]\left(t, x, u(x), u^{(1)}(x), \ldots, u^{(k-1+i)}(x)\right) \\
& =\left(\frac{\partial}{\partial x}\right)^{i}\left[\left[\psi^{+}\right]\left(t, x, u(x), u^{(1)}(x), \ldots, u^{(k-1)}(x)\right)\right] \\
& =\left(\frac{\partial}{\partial x}\right)^{i} \frac{M}{(R-x)^{p}} t^{q}=\frac{p(p+1) \cdots(p+i-1) M}{(R-x)^{p+i}} t^{q} ;
\end{aligned}
$$

by setting $t=r, x=s$ and by using $c=R-s$ we have

$$
D^{i}\left[\psi^{+}\right]\left(r, s, \frac{0 !}{c^{0}}, \frac{1 !}{c^{1}}, \ldots, \frac{(k+i-1) !}{c^{k+i-1}}\right)=\frac{p(p+1) \cdots(p+i-1) M}{c^{p+i}} r^{q} .
$$

Similarly we have

$$
D^{m}\left[\psi^{+}\right]\left(r, s, \frac{0 !}{c^{0}}, \frac{1 !}{c^{1}}, \ldots, \frac{(k+m-1) !}{c^{k+m-1}}\right)=\frac{p(p+1) \cdots(p+m-1) M}{c^{p+m}} r^{q} .
$$


Moreover, by the conditions $0 \leq i \leq m$ and $p \geq 1$ we have

$$
\frac{m !}{i !}=(i+1) \cdots m \leq(p+i) \cdots(p+m-1)=\frac{p(p+1) \cdots(p+m-1)}{p(p+1) \cdots(p+i-1)} .
$$

Thus, we obtain

$$
\begin{aligned}
D^{i}\left[\psi^{+}\right] & \left(r, s, \frac{0 !}{c^{0}}, \frac{1 !}{c^{1}}, \ldots, \frac{(k-1) !}{c^{k-1}}\right) \\
& \leq \frac{i !}{m !} c^{m-i} D^{m}\left[\psi^{+}\right]\left(r, s, \frac{0 !}{c^{0}}, \frac{1 !}{c^{1}}, \ldots, \frac{(k-1) !}{c^{k-1}}\right) .
\end{aligned}
$$

Since in this case $\psi^{+}$is homogeneous of degree $k$ with respect to $\left(t, w_{0}, \ldots\right.$, $\left.w_{k-1}\right)$, this proves the relation

$$
D^{i}\left[\psi^{+}\right]\left(*_{\rho}\right) \ll_{\rho} \frac{i !}{m !} c^{m-i} D^{m}\left[\psi^{+}\right]\left(*_{\rho}\right) .
$$

Thus, (3.4.12) is also proved in the case (ii). This completes the proof of (3) of Lemma 3.4.4.

\subsubsection{On the equation $\left(\tau_{k}-\lambda(x)\right) \phi_{k}=f_{k}$}

For $0<s \leq R$ and

$$
f=\sum_{i+|j|=k} f_{i, j}(x) t^{i} u_{0}^{j_{0}} u_{1}^{j_{1}} \cdots u_{k-1}^{j_{k-1}} \in \mathcal{H}_{k, R}\left[t, u_{0}, \ldots, u_{k-1}\right]
$$

we write $|f|_{s}\left(t, u_{0}, \ldots, u_{k-1}\right)$ by

$$
|f|_{s}\left(t, u_{0}, \ldots, u_{k-1}\right)=\sum_{i+|j|=k}\left\|f_{i, j}\right\|_{s} t^{i} u_{0}^{j_{0}} u_{1}{ }^{j_{1}} \cdots u_{k-1}{ }^{j_{k-1}} .
$$

Let $k \geq 2$, and let us consider the equation

$$
\left(\tau_{k}-\lambda(x)\right) \phi_{k}=f_{k} \in \mathcal{H}_{k, R}\left[t, u_{0}, \ldots, u_{k-1}\right],
$$

where $\tau_{k}$ is the same as in (3.4.2). We have

Lemma 3.4.5. Suppose that

$$
|i+\lambda(x)(j-1)| \geq \sigma_{0}(i+j) \quad \text { on } D_{R} \quad \text { for any } i+j \geq 2
$$

holds for some $\sigma_{0}>0$. Let $k \geq 2$, and let $r_{0}>0$ and $0<c_{0}<R$ be the ones in (3.4.4). Then we have the following results. 
(1) The equation $\left(\tau_{k}-\lambda(x)\right) \phi_{k}=f_{k} \in \mathcal{H}_{k, R}\left[t, u_{0}, \ldots, u_{k-1}\right]$ has a unique solution $\phi_{k} \in \mathcal{H}_{k, R}\left[t, u_{0}, \ldots, u_{k-1}\right]$.

(2) Moreover, if $0<r \leq r_{0}, 0<c \leq c_{0}, 0<s<R, c+s=R$ and $0<\sigma \leq \sigma_{0} / 2$ hold, we have

$$
\begin{aligned}
\sigma k\left|\phi_{k}\right|_{s}( & \left.r \rho, \psi^{+}\left(*_{\rho}\right), D\left[\psi^{+}\right]\left(*_{\rho}\right), \ldots, D^{k-1}\left[\psi^{+}\right]\left(*_{\rho}\right)\right) \\
& \ll_{\rho}\left|f_{k}\right|_{s}\left(r \rho, \psi^{+}\left(*_{\rho}\right), D\left[\psi^{+}\right]\left(*_{\rho}\right), \ldots, D^{k-1}\left[\psi^{+}\right]\left(*_{\rho}\right)\right) .
\end{aligned}
$$

Proof. (1) is clear from (2) of Lemma 3.1.3. Let us show (2). Set

$$
\begin{aligned}
\phi_{k} & =\sum_{i+|j|=k} \phi_{i, j}(x) t^{i} u_{0}^{j_{0}} \cdots u_{k-1}^{j_{k-1}}, \\
f_{k} & =\sum_{i+|j|=k} f_{i, j}(x) t^{i} u_{0}^{j_{0}} \cdots u_{k-1}^{j_{k-1}} .
\end{aligned}
$$

Since the relation $\left(\tau_{k}-\lambda(x)\right) \phi_{k}=f_{k}$ is written in the form (3.1.9), by using (3.4.15) and estimates $\left\|a^{(m)}\right\|_{R} \leq m ! A / \eta^{m}$ and $\left\|\lambda_{m, p}\right\|_{R} \leq(m ! / p !)\left(\Lambda / \eta^{m-p}\right)$ we have

$$
\begin{aligned}
& \sigma_{0} k\left|\phi_{k}\right|_{s}\left(t, u_{0}, \ldots, u_{k-1}\right) \\
& \ll \sum_{i+|j|=k}\left\|\phi_{i, j}\right\|_{s}\left[\sum_{0 \leq m \leq k-1} \frac{m ! A}{\eta^{m}} j_{m} t^{i+1} u_{0} j_{0} \cdots u_{m}^{j_{m}-1} \cdots u_{k-1} j_{k-1}\right] \\
& \quad+\sum_{i+|j|=k}\left\|\phi_{i, j}\right\|_{s}\left[\sum_{1 \leq m \leq k-1} \sum_{0 \leq p \leq m-1} \frac{m !}{p !} \frac{\Lambda}{\eta^{m-p}} j_{m} \times\right. \\
& \left.\times t^{i} u_{0} j_{0} \cdots u_{p}^{j_{p}+1} \cdots u_{m}^{j_{m}-1} \cdots u_{k-1} j_{k-1}\right] \\
& +\left|f_{k}\right|_{s}\left(t, u_{0}, \ldots, u_{k-1}\right)
\end{aligned}
$$

(as formal power series in $\left(t, u_{0}, \ldots, u_{k-1}\right)$ ), and by setting $t=r \rho$ and $u_{p}=$ $D^{p}\left[\psi^{+}\right]\left(*_{\rho}\right)(p=0,1,2, \ldots, k-1)$ we obtain

$$
\begin{array}{r}
\sigma_{0} k\left|\phi_{k}\right|_{s}\left(r \rho, \psi^{+}\left(*_{\rho}\right), D\left[\psi^{+}\right]\left(*_{\rho}\right), \ldots, D^{k-1}\left[\psi^{+}\right]\left(*_{\rho}\right)\right) \\
\ll_{\rho} \sum_{i+|j|=k}\left\|\phi_{i, j}\right\|_{s}\left[\sum_{0 \leq m \leq k-1} \frac{m ! A}{\eta^{m}} j_{m}(r \rho)^{i+1} \psi^{+}\left(*_{\rho}\right)^{j_{0}} \cdots D^{m}\left[\psi^{+}\right]\left(*_{\rho}\right)^{j_{m}-1}\right. \\
\left.\times \cdots \times D^{k-1}\left[\psi^{+}\right]\left(*_{\rho}\right)^{j_{k-1}}\right]
\end{array}
$$




$$
\begin{aligned}
& +\sum_{i+|j|=k}\left\|\phi_{i, j}\right\|_{s}\left[\sum_{1 \leq m \leq k-1} \sum_{0 \leq p \leq m-1} \frac{m !}{p !} \frac{\Lambda}{\eta^{m-p}} j_{m}(r \rho)^{i} \psi^{+}\left(*_{\rho}\right)^{j_{0}} \ldots\right. \\
& \left.\times D^{p}\left[\psi^{+}\right]\left(*_{\rho}\right)^{j_{p}+1} \cdots D^{m}\left[\psi^{+}\right]\left(*_{\rho}\right)^{j_{m}-1} \cdots D^{k-1}\left[\psi^{+}\right]\left(*_{\rho}\right)^{j_{k-1}}\right] \\
& +\left|f_{k}\right|_{s}\left(r \rho, \psi^{+}\left(*_{\rho}\right), D\left[\psi^{+}\right]\left(*_{\rho}\right), \ldots, D^{k-1}\left[\psi^{+}\right]\left(*_{\rho}\right)\right) .
\end{aligned}
$$

Here, we note that by $(2)$ of Lemma 3.4.4 we have $(r \rho)^{i+1} \times D^{m}\left[\psi^{+}\right]\left(*_{\rho}\right)^{j_{m}-1}$ $\ll_{\rho} r\left(c^{m} / m !\right)(\rho r)^{i} \times D^{m}\left[\psi^{+}\right]\left(*_{\rho}\right)^{j_{m}}$ and so

$$
\begin{aligned}
\frac{m ! A}{\eta^{m}} & (r \rho)^{i+1} \psi^{+}\left(*_{\rho}\right)^{j_{0}} \cdots D^{m}\left[\psi^{+}\right]\left(*_{\rho}\right)^{j_{m}-1} \\
& \ll_{\rho} \frac{m ! A}{\eta^{m}} r \frac{c^{m}}{m !} \times\left[(r \rho)^{i} \psi^{+}\left(*_{\rho}\right)^{j_{0}} \cdots D^{m}\left[\psi^{+}\right]\left(*_{\rho}\right)^{j_{m}}\right] \\
& \ll_{\rho} r A \times\left[(r \rho)^{i} \psi^{+}\left(*_{\rho}\right)^{j_{0}} \cdots D^{m}\left[\psi^{+}\right]\left(*_{\rho}\right)^{j_{m}}\right],
\end{aligned}
$$

and by (3) of Lemma 3.4.4 we have $D^{p}\left[\psi^{+}\right]\left(*_{\rho}\right)^{j_{p}+1} \times D^{m}\left[\psi^{+}\right]\left(*_{\rho}\right)^{j_{m}-1} \ll_{\rho}$ $(p ! / m !) c^{m-p} D^{p}\left[\psi^{+}\right]\left(*_{\rho}\right)^{j_{p}} \times D^{m}\left[\psi^{+}\right]\left(*_{\rho}\right)^{j_{m}}$ and so

$$
\begin{aligned}
& \sum_{0 \leq p \leq m-1} \frac{m !}{p !} \frac{\Lambda}{\eta^{m-p}}(r \rho)^{i} \psi^{+}\left(*_{\rho}\right)^{j_{0}} \cdots D^{p}\left[\psi^{+}\right]\left(*_{\rho}\right)^{j_{p}+1} \cdots D^{m}\left[\psi^{+}\right]\left(*_{\rho}\right)^{j_{m}-1} \\
& \quad \ll_{\rho} \sum_{0 \leq p \leq m-1} \frac{m !}{p !} \frac{\Lambda}{\eta^{m-p}} \frac{p !}{m !} c^{m-p} \times\left[(r \rho)^{i} \psi^{+}\left(*_{\rho}\right)^{j_{0}} \cdots D^{m}\left[\psi^{+}\right]\left(*_{\rho}\right)^{j_{m}}\right] \\
& \ll_{\rho}(\Lambda c /(\eta-c))\left[(r \rho)^{i} \psi^{+}\left(*_{\rho}\right)^{j_{0}} \cdots D^{m}\left[\psi^{+}\right]\left(*_{\rho}\right)^{j_{m}}\right] .
\end{aligned}
$$

Hence, by applying these estimates to (3.4.17) and then by using the inequalities $r A+(\Lambda c /(\eta-c)) \leq \sigma_{0} / 2$ (by (3.4.4)) and $j_{0}+j_{1}+j_{2}+\cdots+j_{k-1}=|j| \leq k$ we obtain

$$
\begin{aligned}
& \sigma_{0} k\left|\phi_{k}\right|_{s}\left(r \rho, \psi^{+}\left(*_{\rho}\right), D\left[\psi^{+}\right]\left(*_{\rho}\right), \ldots, D^{k-1}\left[\psi^{+}\right]\left(*_{\rho}\right)\right) \\
& \ll_{\rho} \sum_{i+|j|=k}\left\|\phi_{i, j}\right\|_{s}\left[\sum_{0 \leq m \leq k-1} r A j_{m} \times(r \rho)^{i} \psi^{+}\left(*_{\rho}\right)^{j_{0}} \cdots D^{k-1}\left[\psi^{+}\right]\left(*_{\rho}\right)^{j_{k-1}}\right. \\
& \left.\quad+\sum_{1 \leq m \leq k-1}(\Lambda c /(\eta-c)) j_{m} \times(r \rho)^{i} \psi^{+}\left(*_{\rho}\right)^{j_{0}} \cdots D^{k-1}\left[\psi^{+}\right]\left(*_{\rho}\right)^{j_{k-1}}\right] \\
& \quad+\left|f_{k}\right|_{s}\left(r \rho, \psi^{+}\left(*_{\rho}\right), D\left[\psi^{+}\right]\left(*_{\rho}\right), \ldots, D^{k-1}\left[\psi^{+}\right]\left(*_{\rho}\right)\right)
\end{aligned}
$$




$$
\begin{aligned}
& =\sum_{i+|j|=k}\left\|\phi_{i, j}\right\|_{s}(r A+(\Lambda c /(\eta-c)))\left[\sum_{0 \leq m \leq k-1} j_{m}\right] \times \\
& \times(r \rho)^{i} \psi^{+}\left(*_{\rho}\right)^{j_{0}} \cdots D^{k-1}\left[\psi^{+}\right]\left(*_{\rho}\right)^{j_{k-1}} \\
& +\left|f_{k}\right|_{s}\left(r \rho, \psi^{+}\left(*_{\rho}\right), D\left[\psi^{+}\right]\left(*_{\rho}\right), \ldots, D^{k-1}\left[\psi^{+}\right]\left(*_{\rho}\right)\right) \\
& \ll_{\rho} \sum_{i+|j|=k}\left\|\phi_{i, j}\right\|_{s}\left(\sigma_{0} / 2\right) k \times(r \rho)^{i} \psi^{+}\left(*_{\rho}\right)^{j_{0}} \cdots D^{k-1}\left[\psi^{+}\right]\left(*_{\rho}\right)^{j_{k-1}} \\
& +\left|f_{k}\right|_{s}\left(r \rho, \psi^{+}\left(*_{\rho}\right), D\left[\psi^{+}\right]\left(*_{\rho}\right), \ldots, D^{k-1}\left[\psi^{+}\right]\left(*_{\rho}\right)\right) \\
& =\frac{\sigma_{0}}{2} k\left|\phi_{k}\right|_{s}\left(r \rho, \psi^{+}\left(*_{\rho}\right), D\left[\psi^{+}\right]\left(*_{\rho}\right), \ldots, D^{k-1}\left[\psi^{+}\right]\left(*_{\rho}\right)\right) \\
& +\left|f_{k}\right|_{s}\left(r \rho, \psi^{+}\left(*_{\rho}\right), D\left[\psi^{+}\right]\left(*_{\rho}\right), \ldots, D^{k-1}\left[\psi^{+}\right]\left(*_{\rho}\right)\right) .
\end{aligned}
$$

Thus we have proved the inequality

$$
\begin{aligned}
\frac{\sigma_{0}}{2} k\left|\phi_{k}\right|_{s} & \left(r \rho, \psi^{+}\left(*_{\rho}\right), D\left[\psi^{+}\right]\left(*_{\rho}\right), \ldots, D^{k-1}\left[\psi^{+}\right]\left(*_{\rho}\right)\right) \\
& \ll_{\rho}\left|f_{k}\right|_{s}\left(r \rho, \psi^{+}\left(*_{\rho}\right), D\left[\psi^{+}\right]\left(*_{\rho}\right), \ldots, D^{k-1}\left[\psi^{+}\right]\left(*_{\rho}\right)\right) .
\end{aligned}
$$

This proves the part (2) Lemma 3.4.5.

\subsubsection{Proof of Proposition 3.4.3}

We will give a proof of Proposition 3.4.3 from now. The essential part of the proof consists of two propositions (Proposition 3.4.6 and Proposition 3.4.8) given below.

Proposition 3.4.6. Let $\psi^{+}=\sum_{p \geq 1} \psi_{p}^{+}\left(t, x, w_{0}, \ldots, w_{p-1}\right)$ be the formal solution of (3.4.6) with $\psi_{1}^{+}=w_{0}$.

(1) We have the following equalities:

$$
\begin{aligned}
& G\left(t, x, \psi^{+}, D\left[\psi^{+}\right]\right)=\psi_{2}^{+}+2 \psi_{3}^{+}+3 \psi_{4}^{+}+\cdots=\sum_{p \geq 1}(p-1) \psi_{p}^{+}, \\
& D^{m}[G]\left(t, x, \psi^{+}, \cdots, D^{m+1}\left[\psi^{+}\right]\right)=\sum_{p \geq 1}(p-1) D^{m}\left[\psi_{p}^{+}\right] .
\end{aligned}
$$

(2) Let $\varphi\left(t, x, u_{0}, \ldots, u_{k-1}\right) \in \mathcal{H}_{k, R}\left[t, u_{0}, \ldots, u_{k-1}\right]$. If

$$
\begin{aligned}
& \varphi\left(t, x, \psi^{+}, D\left[\psi^{+}\right], \ldots, D^{k-1}\left[\psi^{+}\right]\right) \\
& =\sum_{p \geq k} h_{p}\left(t, x, w_{0}, \ldots, w_{p-1}\right) \in \sum_{p \geq k} \mathcal{H}_{p,(R)}\left[t, w_{0}, \ldots, w_{p-1}\right]
\end{aligned}
$$


holds, we have

$$
\begin{gathered}
\sum_{0 \leq m \leq k-1} D^{m}[G]\left(t, x, \psi^{+}, D\left[\psi^{+}\right], \cdots, D^{m+1}\left[\psi^{+}\right]\right) \times \\
\times \frac{\partial \varphi}{\partial u_{m}}\left(t, x, \psi^{+}, D\left[\psi^{+}\right], \ldots, D^{k-1}\left[\psi^{+}\right]\right) \\
=\sum_{p \geq k}(p-k) h_{p}\left(t, x, w_{0}, \ldots, w_{p-1}\right) \in \sum_{p \geq k} \mathcal{H}_{p,(R)}\left[t, w_{0}, \ldots, w_{p-1}\right] .
\end{gathered}
$$

(3) Let $\varphi\left(t, x, u_{0}, \ldots, u_{k-1}\right) \in \mathcal{H}_{k, R}\left[t, u_{0}, \ldots, u_{k-1}\right]$. If $c>0,0<s<R$, and

$$
|\varphi|_{s}\left(r \rho, \psi^{+}\left(*_{\rho}\right), D\left[\psi^{+}\right]\left(*_{\rho}\right), \ldots, D^{k-1}\left[\psi^{+}\right]\left(*_{\rho}\right)\right) \ll_{\rho} \sum_{p \geq k} B_{p} \rho^{p}
$$

holds for some $B_{p} \geq 0(p \geq k)$, we have

$$
\begin{aligned}
& \sum_{0 \leq m \leq k-1} D^{m}[G]\left(r \rho, s, \psi^{+}\left(*_{\rho}\right), D\left[\psi^{+}\right]\left(*_{\rho}\right), \cdots, D^{m+1}\left[\psi^{+}\right]\left(*_{\rho}\right)\right) \times \\
& \quad \times \frac{\partial|\varphi|_{s}}{\partial u_{m}}\left(r \rho, \psi^{+}\left(*_{\rho}\right), D\left[\psi^{+}\right]\left(*_{\rho}\right), \ldots, D^{k-1}\left[\psi^{+}\right]\left(*_{\rho}\right)\right) \\
& \ll \rho \sum_{p \geq k}(p-k) B_{p} \rho^{p} .
\end{aligned}
$$

Proof. For a function $K\left(t, x, u_{0}, \ldots, u_{k-1}\right)$ we write $K^{*}=K^{*}\left(t, x, w_{0}\right.$, $\left.w_{1}, \ldots\right)=K\left(t, x, \psi^{+}, D\left[\psi^{+}\right], \ldots, D^{k-1}\left[\psi^{+}\right]\right)$.

Since $\tau^{0}$ has the form $\tau^{0}=t \partial / \partial t+\sum_{m \geq 0} w_{m} \partial / \partial w_{m}$, by (3.4.6) and the Euler's identity we have

$$
G^{*}=\left(\tau^{0}-1\right) \psi^{+}=\sum_{p \geq 1}\left(\tau^{0}-1\right) \psi_{p}^{+}=\sum_{p \geq 1}(p-1) \psi_{p}^{+}:
$$

this proves (3.4.18). Moreover, by Lemma 2.5 and (3.4.18) we can verify the equality (3.4.19) in the following way:

$$
\left(D^{m}[G]\right)^{*}=D^{m}\left[G^{*}\right]=D^{m}\left[\sum_{p \geq 1}(p-1) \psi_{p}^{+}\right]=\sum_{p \geq 1}(p-1) D^{m}\left[\psi_{p}^{+}\right] .
$$

Let us show (2). By applying $\tau^{0}$ to the both sides of (3.4.20) we have

$$
\begin{aligned}
& \sum_{p \geq k} p h_{p}\left(t, x, w_{0}, \ldots, w_{p-1}\right)=\tau^{0} \sum_{p \geq k} h_{p}\left(t, x, w_{0}, \ldots, w_{p-1}\right) \\
& =\tau^{0} \varphi\left(t, x, \psi^{+}, D\left[\psi^{+}\right], \ldots, D^{k-1}\left[\psi^{+}\right]\right)
\end{aligned}
$$




$$
\begin{aligned}
=\left(t \frac{\partial \varphi}{\partial t}\right)^{*}+ & \sum_{0 \leq m \leq k-1}\left(\frac{\partial \varphi}{\partial u_{m}}\right)^{*} \times t \frac{\partial}{\partial t} D^{m}\left[\psi^{+}\right] \\
& +\sum_{i \geq 0} \sum_{0 \leq m \leq k-1}\left(\frac{\partial \varphi}{\partial u_{m}}\right)^{*} \times w_{i} \frac{\partial}{\partial w_{i}} D^{m}\left[\psi^{+}\right] \\
=\left(t \frac{\partial \varphi}{\partial t}\right)^{*}+ & \sum_{0 \leq m \leq k-1}\left(\frac{\partial \varphi}{\partial u_{m}}\right)^{*} \times \tau^{0} D^{m}\left[\psi^{+}\right] .
\end{aligned}
$$

On the other hand, since $\varphi\left(t, x, u_{0}, \ldots, u_{k-1}\right)$ is homogeneous of degree $k$ with respect to $\left(t, u_{0}, \ldots, u_{k-1}\right)$, by the Euler's identity we have

$$
k \varphi\left(t, x, u_{0}, \ldots, u_{k-1}\right)=\tau^{0} \varphi\left(t, x, u_{0}, \ldots, u_{k-1}\right)=t \frac{\partial \varphi}{\partial t}+\sum_{0 \leq m \leq k-1} u_{m} \frac{\partial \varphi}{\partial u_{m}}
$$

and so by (3.4.20) we have

$$
\begin{aligned}
& \sum_{p \geq k} k h_{p}\left(t, x, w_{0}, \ldots, w_{p-1}\right)=k \varphi\left(t, x, \psi^{+}, D\left[\psi^{+}\right], \ldots, D^{k-1}\left[\psi^{+}\right]\right) \\
& =\left(t \frac{\partial \varphi}{\partial t}\right)^{*}+\sum_{0 \leq m \leq k-1} D^{m}\left[\psi^{+}\right] \times\left(\frac{\partial \varphi}{\partial u_{m}}\right)^{*} .
\end{aligned}
$$

Hence, by (3.4.24) and (3.4.25) we have

$$
\begin{aligned}
& \sum_{p \geq k}(p-k) h_{p}\left(t, x, w_{0}, \ldots, w_{p-1}\right) \\
&=\sum_{0 \leq m \leq k-1}\left(\tau^{0}-1\right) D^{m}\left[\psi^{+}\right] \times\left(\frac{\partial \varphi}{\partial u_{m}}\right)^{*} .
\end{aligned}
$$

Here we note:

Lemma 3.4.7. For any function $f\left(t, x, w_{0}, w_{1}, \ldots\right)$ we have

$$
\left(\tau^{0} \circ D\right) f\left(t, x, w_{0}, w_{1}, \ldots\right)=\left(D \circ \tau^{0}\right) f\left(t, x, w_{0}, w_{1}, \ldots\right) .
$$

Proof of Lemma 3.4.7. Since

$$
\begin{aligned}
\left(\tau^{0} \circ D\right) f-\left(D \circ \tau^{0}\right) f=\left(t \frac{\partial}{\partial t}\right. & \left.+\sum_{j \geq 0} w_{j} \frac{\partial}{\partial w_{j}}\right)\left(\frac{\partial f}{\partial x}+\sum_{i \geq 0} w_{i+1} \frac{\partial f}{\partial w_{i}}\right) \\
& -\left(\frac{\partial}{\partial x}+\sum_{i \geq 0} w_{i+1} \frac{\partial}{\partial w_{i}}\right)\left(t \frac{\partial f}{\partial t}+\sum_{j \geq 0} w_{j} \frac{\partial f}{\partial w_{j}}\right),
\end{aligned}
$$

we can verify (3.4.27) by a direct calculation. 
Hence, by applying this lemma to (3.4.26) we have

$$
\begin{aligned}
\sum_{p \geq k}(p-k) h_{p}\left(t, x, w_{0}, \ldots, w_{p-1}\right) & \\
& =\sum_{0 \leq m \leq k-1} D^{m}\left[\left(\tau^{0}-1\right)\left(\psi^{+}\right)\right] \times\left(\frac{\partial \varphi}{\partial u_{m}}\right)^{*} \\
& =\sum_{0 \leq m \leq k-1} D^{m}\left[G\left(t, x, \psi^{+}, D\left[\psi^{+}\right]\right)\right] \times\left(\frac{\partial \varphi}{\partial u_{m}}\right)^{*} \\
& =\sum_{0 \leq m \leq k-1} D^{m}[G]\left(t, x, \psi^{+}, \ldots, D^{m+1}\left[\psi^{+}\right]\right) \times\left(\frac{\partial \varphi}{\partial u_{m}}\right)^{*} .
\end{aligned}
$$

This proves (3.4.21): the part (2) of Proposition 3.4.6 is proved.

Let us show (3). Let $\varphi\left(t, x, u_{0}, \ldots, u_{k-1}\right) \in \mathcal{H}_{k, R}\left[t, u_{0}, \ldots, u_{k-1}\right]$. Since $|\varphi|_{s}\left(t, u_{0}, \ldots, u_{k-1}\right) \in \mathcal{H}_{k, R}\left[t, u_{0}, \ldots, u_{k-1}\right]$ holds, we can set

$$
\begin{aligned}
& |\varphi|_{s}\left(t, \psi^{+}, D\left[\psi^{+}\right], \ldots, D^{k-1}\left[\psi^{+}\right]\right) \\
& =\sum_{p \geq k} h_{p}\left(t, x, w_{0}, \ldots, w_{p-1}\right) \in \sum_{p \geq k} \mathcal{H}_{p,(R)}\left[t, w_{0}, \ldots, w_{p-1}\right]
\end{aligned}
$$

and by the part (2) of this proposition we have

$$
\begin{aligned}
& \sum_{0 \leq m \leq k-1} D^{m}[G]\left(t, x, \psi^{+}, D\left[\psi^{+}\right], \cdots, D^{m+1}\left[\psi^{+}\right]\right) \times \\
& \times \frac{\partial|\varphi|_{s}}{\partial u_{m}}\left(t, \psi^{+}, D\left[\psi^{+}\right], \ldots, D^{k-1}\left[\psi^{+}\right]\right) \\
& =\sum_{p \geq k}(p-k) h_{p}\left(t, x, w_{0}, \ldots, w_{p-1}\right) \in \sum_{p \geq k} \mathcal{H}_{p,(R)}\left[t, w_{0}, \ldots, w_{p-1}\right] .
\end{aligned}
$$

By setting $t=r \rho, x=s$ and $w_{p}=\left(p ! / c^{p}\right) \rho(p=0,1,2, \ldots)$ in (3.4.28) and (3.4.29) we have

$$
\begin{aligned}
& |\varphi|_{s}\left(r \rho, \psi^{+}\left(*_{\rho}\right), D\left[\psi^{+}\right]\left(*_{\rho}\right), \ldots, D^{k-1}\left[\psi^{+}\right]\left(*_{\rho}\right)\right) \\
& =\sum_{p \geq k} h_{p}(*) \rho^{p} \in \mathbb{C}[[\rho]], \\
& \sum_{0 \leq m \leq k-1} D^{m}[G]\left(r \rho, s, \psi^{+}\left(*_{\rho}\right), D\left[\psi^{+}\right]\left(*_{\rho}\right), \cdots, D^{m+1}\left[\psi^{+}\right]\left(*_{\rho}\right)\right) \times \\
& \quad \times \frac{\partial|\varphi|_{s}}{\partial u_{m}}\left(r \rho, \psi^{+}\left(*_{\rho}\right), D\left[\psi^{+}\right]\left(*_{\rho}\right), \ldots, D^{k-1}\left[\psi^{+}\right]\left(*_{\rho}\right)\right) \\
& =\sum_{p \geq k}(p-k) h_{p}(*) \rho^{p} \in \mathbb{C}[[\rho]],
\end{aligned}
$$


where

$$
(*)=\left(r, s, \frac{0 !}{c^{0}}, \frac{1 !}{c^{1}}, \frac{2 !}{c^{2}}, \ldots\right) .
$$

Hence, by comparing (3.4.22) and (3.4.30) we have $h_{p}(*) \leq B_{p}$ for $p \geq k$, and by applying this to the right-hand side of (3.4.31) we obtain the majorant relation (3.4.23).

Let $\phi=\sum_{k>1} \phi_{k}\left(t, x, u_{0}, \ldots, u_{k-1}\right)$ be the formal solution (3.4.1) with $\phi_{1}=-\alpha(x) \beta(x) t+\beta(x) u_{0}$ and $\phi_{p}\left(t, x, u_{0}, \ldots, u_{k-1}\right) \in \mathcal{H}_{k, R}\left[t, u_{0}, \ldots, u_{k-1}\right]$ (for $k \geq 2$ ).

Proposition 3.4.8. Suppose the conditions (3.4.3) and (3.4.4). Then, we have the following results:

(1) For any $r>0, c>0$ and $0<s<R$, we have

$$
\begin{aligned}
& \left|\phi_{1}\right|_{s}\left(r \rho, \psi^{+}\left(*_{\rho}\right)\right) \\
& \quad \ll_{\rho}\|\beta\|_{s}\left(\|\alpha\|_{s} r \rho+\psi_{1}^{+}(*) \rho+\psi_{2}^{+}(*) \rho^{2}+\psi_{3}^{+}(*) \rho^{3}+\cdots\right) .
\end{aligned}
$$

(2) For any $k \geq 2,0<r \leq r_{0}, 0<c \leq c_{0}, 0<s<R$ satisfying $c+s=R$ and $0<\sigma \leq \min \left\{1, \sigma_{0} / 2\right\}$ we have

$$
\begin{array}{r}
(3.4 .33)_{k} \quad \sum_{1 \leq q \leq k-1} \sum_{0 \leq m \leq q-1} D^{m}[G]\left(r \rho, s, \psi^{+}\left(*_{\rho}\right), \cdots, D^{m+1}\left[\psi^{+}\right]\left(*_{\rho}\right)\right) \times \\
\times \frac{\partial\left|\phi_{q}\right|_{s}}{\partial u_{m}}\left(r \rho, \psi^{+}\left(*_{\rho}\right), \ldots, D^{q-1}\left[\psi^{+}\right]\left(*_{\rho}\right)\right) \\
\ll_{\rho}\|\beta\|_{s} \sum_{2 \leq p \leq k-1} \frac{(p-1)^{p-1}}{\sigma^{p-2}(p-2) !} \psi_{p}^{+}(*) \rho^{p} \\
+\|\beta\|_{s} \sum_{p \geq k} \frac{(p-1)^{k-1}}{\sigma^{k-2}(k-2) !} \psi_{p}^{+}(*) \rho^{p}, \\
(3.4 .34)_{k} \quad\left|\phi_{k}\right|_{s}\left(r \rho, \psi^{+}\left(*_{\rho}\right), \ldots, D^{k-1}\left[\psi^{+}\right]\left(*_{\rho}\right)\right) \\
\ll_{\rho}\|\beta\|_{s} \sum_{p \geq k} \frac{(p-1)^{k-1}}{\sigma^{k-1}(k-1) !} \psi_{p}^{+}(*) \rho^{p} .
\end{array}
$$

Proof. Since $\left|\phi_{1}\right|_{s}\left(t, u_{0}\right)=\|\alpha \beta\|_{s} t+\|\beta\|_{s} u_{0}$, we have

$$
\begin{aligned}
\left|\phi_{1}\right|_{s}\left(r \rho, \psi^{+}\left(*_{\rho}\right)\right) & =\|\alpha \beta\|_{s} r \rho+\|\beta\|_{s} \psi^{+}\left(*_{\rho}\right) \\
& \ll_{\rho}\|\beta\|_{s}\left(\|\alpha\|_{s} r \rho+\psi^{+}\left(*_{\rho}\right)\right) \\
& =\|\beta\|_{s}\left(\|\alpha\|_{s} r \rho+\psi_{1}^{+}(*) \rho+\psi_{2}^{+}(*) \rho^{2}+\psi_{3}^{+}(*) \rho^{3}+\cdots\right) ;
\end{aligned}
$$


this proves the part (1).

Let us show (2) by induction on $k$. The condition $(3.4 .33)_{2}$ is verified by (3.4.18) and

$$
\begin{aligned}
& G\left(r \rho, s, \psi^{+}\left(*_{\rho}\right), D\left[\psi^{+}\right]\left(*_{\rho}\right)\right) \frac{\partial\left|\phi_{1}\right|_{s}}{\partial u_{0}}=G\left(r \rho, s, \psi^{+}\left(*_{\rho}\right), D\left[\psi^{+}\right]\left(*_{\rho}\right)\right)\|\beta\|_{s} \\
& =\|\beta\|_{s}\left(\psi_{2}^{+}(*) \rho^{2}+2 \psi_{3}^{+}(*) \rho^{3}+\cdots\right)=\|\beta\|_{s} \sum_{p \geq 2}(p-1) \psi_{p}^{+}(*) \rho^{p} .
\end{aligned}
$$

By (3.4.2) we have

$$
\left(\tau_{2}-\lambda(x)\right) \phi_{2}=-R_{2}\left(t, x, u_{0}, u_{1}\right) \frac{\partial \phi_{1}}{\partial u_{0}}=-R_{2}\left(t, x, u_{0}, u_{1}\right) \beta(x) ;
$$

by applying (2) of Lemma 3.4.5 to this equation and by using the majorant relation $R_{2}\left(t, x, u_{0}, u_{1}\right) \ll G\left(t, x, u_{0}, u_{1}\right)$ (by (3.4.5)) we have

$$
\begin{aligned}
& 2 \sigma\left|\phi_{2}\right|_{s}(\left(r \rho, \psi^{+}\left(*_{\rho}\right), D\left[\psi^{+}\right]\left(*_{\rho}\right)\right) \\
& \ll_{\rho}\|\beta\|_{s}\left|R_{2}\right|_{s}\left(r \rho, \psi^{+}\left(*_{\rho}\right), D\left[\psi^{+}\right]\left(*_{\rho}\right)\right) \\
& \ll_{\rho}\|\beta\|_{s} G\left(r \rho, s, \psi^{+}\left(*_{\rho}\right), D\left[\psi^{+}\right]\left(*_{\rho}\right)\right) .
\end{aligned}
$$

Here, we used the following fact: if $f(x) \ll g(x)$ holds we have $\|f\|_{s} \leq g(s)$, and so we have $\left|R_{2}\right|_{s}\left(t, u_{0}, u_{1}\right) \ll G\left(t, s, u_{0}, u_{1}\right)$ (as power series in $\left(t, u_{0}, u_{1}\right)$ ). Thus, the condition $(3.4 .34)_{2}$ is verified by (3.4.18) and

$$
\begin{aligned}
\left|\phi_{2}\right|_{s} & \left(r \rho, \psi^{+}\left(*_{\rho}\right), D\left[\psi^{+}\right]\left(*_{\rho}\right)\right) \\
& \ll_{\rho} \frac{1}{2 \sigma}\|\beta\|_{s} G\left(r \rho, s, \psi^{+}\left(*_{\rho}\right), D\left[\psi^{+}\right]\left(*_{\rho}\right)\right) \\
& \ll_{\rho} \frac{1}{\sigma}\|\beta\|_{s}\left(\psi_{2}^{+}(*) \rho^{2}+2 \psi_{3}^{+}(*) \rho^{3}+\cdots\right)=\|\beta\|_{s} \sum_{p \geq 2} \frac{(p-1)}{\sigma} \psi_{p}^{+}(*) \rho^{p} .
\end{aligned}
$$

Now, let $k \geq 3$ and suppose that $(3.4 .33)_{k-1}$ and (3.4.34) $k-1$ are already proved. Note

$$
\begin{aligned}
& \sum_{1 \leq q \leq k-1} \sum_{0 \leq m \leq q-1} D^{m}[G] \frac{\partial\left|\phi_{q}\right|_{s}}{\partial u_{m}} \\
= & \sum_{1 \leq q \leq k-2} \sum_{0 \leq m \leq q-1} D^{m}[G] \frac{\partial\left|\phi_{q}\right|_{s}}{\partial u_{m}}+\sum_{0 \leq m \leq k-2} D^{m}[G] \frac{\partial\left|\phi_{k-1}\right|_{s}}{\partial u_{m}} .
\end{aligned}
$$

To the first sum of the right-hand side we can apply the induction hypothesis $(3.4 .33)_{k-1}$, and to the second sum we can apply the induction hypothesis 
(3.4.34) $)_{k-1}$ and (3) of Proposition 3.4.6 (with $\varphi$ replaced by $\phi_{k-1}$ ): thus we have

$$
\begin{aligned}
& \sum_{1 \leq q \leq k-1} \sum_{0 \leq m \leq q-1} D^{m}[G]\left(r \rho, s, \psi^{+}\left(*_{\rho}\right), \cdots, D^{m+1}\left[\psi^{+}\right]\left(*_{\rho}\right)\right) \times \\
& \quad \times \frac{\partial\left|\phi_{q}\right|_{s}}{\partial u_{m}}\left(r \rho, \psi^{+}\left(*_{\rho}\right), D\left[\psi^{+}\right]\left(*_{\rho}\right), \ldots, D^{q-1}\left[\psi^{+}\right]\left(*_{\rho}\right)\right) \\
& \ll_{\rho}\|\beta\|_{s} \sum_{2 \leq p \leq k-2} \frac{(p-1)^{p-1}}{\sigma^{p-2}(p-2) !} \psi_{p}^{+}(*) \rho^{p}+\|\beta\|_{s} \sum_{p \geq k-1} \frac{(p-1)^{k-2}}{\sigma^{k-3}(k-3) !} \psi_{p}^{+}(*) \rho^{p} \\
& \quad+\|\beta\|_{s} \sum_{p \geq k-1}(p-k+1) \frac{(p-1)^{k-2}}{\sigma^{k-2}(k-2) !} \psi_{p}^{+}(*) \rho^{p} \\
& =\|\beta\|_{s} \sum_{2 \leq p \leq k-1} \frac{(p-1)^{p-1}}{\sigma^{p-2}(p-2) !} \psi_{p}^{+}(*) \rho^{p} \\
& \quad+\|\beta\|_{s} \sum_{p \geq k}\left(\frac{(p-1)^{k-2}}{\sigma^{k-3}(k-3) !}+\frac{(p-k+1)(p-1)^{k-2}}{\sigma^{k-2}(k-2) !}\right) \psi_{p}^{+}(*) \rho^{p} .
\end{aligned}
$$

Since $0<\sigma \leq 1$ is assumed and since

$$
\begin{aligned}
& \frac{(p-1)^{k-2}}{\sigma^{k-3}(k-3) !}+\frac{(p-k+1)(p-1)^{k-2}}{\sigma^{k-2}(k-2) !} \\
& \leq \frac{(p-1)^{k-2}}{\sigma^{k-2}(k-2) !}((k-2)+(p-k+1))=\frac{(p-1)^{k-1}}{\sigma^{k-2}(k-2) !},
\end{aligned}
$$

by (3.4.35) we obtain $(3.4 .33)_{k}$.

Let us show $(3.4 .34)_{k}$. Since $R_{k-q+1}\left(t, x, u_{0}, u_{1}\right) \ll G\left(t, x, u_{0}, u_{1}\right)$ (by (3.4.5)) we have $D^{m}\left[R_{k-q+1}\right]\left(t, x, u_{0}, \ldots, u_{m+1}\right) \ll D^{m}[G]\left(t, x, u_{0}, \ldots, u_{m+1}\right)$ and so

$$
\left|D^{m}\left[R_{k-q+1}\right]\right|_{s}\left(t, u_{0}, \ldots, u_{m+1}\right) \ll D^{m}[G]\left(t, s, u_{0}, \ldots, u_{m+1}\right)
$$

as power series in $\left(t, u_{0}, \ldots, u_{m+1}\right)$. By using this fact, (3.4.2), (2) of Lemma 3.4 .5 and the relation

$$
\left|\frac{\partial \phi_{q}}{\partial u_{m}}\right|_{s}\left(t, u_{0}, \ldots, u_{q-1}\right)=\frac{\partial\left|\phi_{q}\right|_{s}}{\partial u_{m}}\left(t, u_{0}, \ldots, u_{q-1}\right)
$$


we have

$$
\begin{aligned}
& \left|\phi_{k}\right|_{s}\left(r \rho, \psi^{+}\left(*_{\rho}\right), \ldots, D^{k-1}\left[\psi^{+}\right]\left(*_{\rho}\right)\right) \\
& \ll_{\rho} \frac{1}{\sigma k} \sum_{1 \leq q \leq k-1} \sum_{0 \leq m \leq q-1}\left|D^{m}\left[R_{k-q+1}\right]\right|_{s}\left(r \rho, \psi^{+}\left(*_{\rho}\right), \ldots, D^{m+1}\left[\psi^{+}\right]\left(*_{\rho}\right)\right) \\
& \times \frac{\partial\left|\phi_{q}\right|_{s}}{\partial u_{m}}\left(r \rho, \psi^{+}\left(*_{\rho}\right), \ldots, D^{q-1}\left[\psi^{+}\right]\left(*_{\rho}\right)\right) \\
& \ll_{\rho} \frac{1}{\sigma(k-1)} \sum_{1 \leq q \leq k-1} \sum_{0 \leq m \leq q-1} D^{m}[G]\left(r \rho, s, \psi^{+}\left(*_{\rho}\right), \ldots, D^{m+1}\left[\psi^{+}\right]\left(*_{\rho}\right)\right) \\
& \times \frac{\partial\left|\phi_{q}\right|_{s}}{\partial u_{m}}\left(r \rho, \psi^{+}\left(*_{\rho}\right), \ldots, D^{q-1}\left[\psi^{+}\right]\left(*_{\rho}\right)\right) .
\end{aligned}
$$

Therefore, by applying $(3.4 .33)_{k}$ to this inequality we have

$$
\begin{gathered}
\left|\phi_{k}\right|_{s}\left(r \rho, \psi^{+}\left(*_{\rho}\right), \ldots, D^{k-1}\left[\psi^{+}\right]\left(*_{\rho}\right)\right) \\
\ll_{\rho}\|\beta\|_{s} \sum_{2 \leq p \leq k-1} \frac{(p-1)^{p-1}}{\sigma^{p-1}(k-1)(p-2) !} \psi_{p}^{+}(*) \rho^{p} \\
+\|\beta\|_{s} \sum_{p \geq k} \frac{(p-1)^{k-1}}{\sigma^{k-1}(k-1) !} \psi_{p}^{+}(*) \rho^{p} .
\end{gathered}
$$

Since the degree of the left-hand side with respect to $\rho$ is greater than or equal to $k$ we obtain

$$
\left|\phi_{k}\right|_{s}\left(r \rho, \psi^{+}\left(*_{\rho}\right), \ldots, D^{k-1}\left[\psi^{+}\right]\left(*_{\rho}\right)\right) \ll_{\rho}\|\beta\|_{s} \sum_{p \geq k} \frac{(p-1)^{k-1}}{\sigma^{k-1}(k-1) !} \psi_{p}^{+}(*) \rho^{p}:
$$

this proves $(3.4 .34)_{k}$.

Proof of Proposition 3.4.3. By comparing the coefficients of $\rho$ in the both sides of (3.4.32) we have

$$
\left|\phi_{1}\right|_{s}\left(r, \psi_{1}^{+}(*)\right) \leq\|\beta\|_{s}\left(\|\alpha\|_{s} r+\psi_{1}^{+}(*)\right):
$$

this implies the inequality (3.4.9). Note that $\phi_{1}=-\alpha(x) \beta(x) t+\beta(x) u_{0}$ and $\psi_{1}^{+}=w_{0}$ hold.

Similarly, by comparing the homogeneous part of degree $k$ with respect to $\rho$ in the both sides of $(3.4 .34)_{k}$ we have

$$
\left|\phi_{k}\right|_{s}\left(r, \psi_{1}^{+}(*), \ldots, D^{k-1}\left[\psi_{1}^{+}\right](*)\right) \leq\|\beta\|_{s} \frac{(k-1)^{k-1}}{\sigma^{k-1}(k-1) !} \psi_{k}^{+}(*)
$$


for any $k \geq 2$. Since $D^{j}\left[\psi_{1}^{+}\right](*)=\left(D^{j}\left[w_{0}\right]\right)(*)=\left(w_{j}\right)(*)=j ! / c^{j}$ holds for all $j \in \mathbb{N}$, we obtain

$$
\begin{aligned}
& \left|\phi_{k}\right|_{s}\left(r, \frac{0 !}{c^{0}}, \frac{1 !}{c^{1}}, \ldots, \frac{(k-1) !}{c^{k-1}}\right) \\
& \quad \leq\|\beta\|_{s} \frac{(k-1)^{k-1}}{\sigma^{k-1}(k-1) !} \psi_{k}^{+}\left(r, s, \frac{0 !}{c^{0}}, \frac{1 !}{c^{1}}, \ldots, \frac{(k-1) !}{c^{k-1}}\right) .
\end{aligned}
$$

Since $\left|\psi_{k}^{+}\right|_{s}\left(t, w_{0}, \ldots, w_{k-1}\right)=\psi_{k}^{+}\left(t, s, w_{0}, \ldots, w_{k-1}\right)$ holds, we have the inequality (3.4.10).

\section{§3.5. Equivalence of two PDEs}

Let us find suitable function-spaces $\mathcal{F}$ and $\mathcal{G}$ so that the following two partial differential equations

$$
\begin{aligned}
t \frac{\partial u}{\partial t} & =F\left(t, x, u, \frac{\partial u}{\partial x}\right) \quad \text { in } \mathcal{F}, \\
t \frac{\partial w}{\partial t} & =\lambda(x) w \text { in } \mathcal{G}
\end{aligned}
$$

are equivalent in the sense defined below (in Definition 3.5.1). Set

$$
\begin{aligned}
& \mathcal{S}_{A}=\text { the set of all solutions of (3.5.1) in } \mathcal{F}, \\
& \mathcal{S}_{B}=\text { the set of all solutions of (3.5.2) in } \mathcal{G} .
\end{aligned}
$$

Definition 3.5.1. Let $\phi\left(t, x, u_{0}, u_{1}, \ldots\right)$ and $\psi\left(t, x, w_{0}, w_{1}, \ldots\right)$ be solutions of $(\Phi)$ and $(\Psi)$, respectively. We say that the two equations (3.5.1) and (3.5.2) are equivalent, if the two mappings

$$
\begin{aligned}
& \Phi: \mathcal{S}_{A} \ni u(t, x) \longmapsto w(t, x)=\phi(t, x, u, \partial u / \partial x, \ldots) \in \mathcal{S}_{B}, \\
& \Psi: \mathcal{S}_{B} \ni w(t, x) \longmapsto u(t, x)=\psi(t, x, w, \partial w / \partial x, \ldots) \in \mathcal{S}_{A}
\end{aligned}
$$

are well-defined, bijective and one is the inverse of the other.

$$
\begin{aligned}
& \text { For } r>0, c>0, s>0 \text { and } \varepsilon>0 \text { we write } \\
& \qquad \begin{aligned}
U_{k}(r, c, s, \varepsilon)= & \left\{\left(t, x, u_{0}, \ldots, u_{k-1}\right) \in \mathbb{C} \times \mathbb{C} \times \mathbb{C}^{k} ;|t| \leq r \varepsilon,|x| \leq s,\right. \\
& \left.\left|u_{0}\right| \leq 0 ! \varepsilon,\left|u_{1}\right| \leq 1 ! \varepsilon / c, \ldots,\left|u_{k-1}\right| \leq(k-1) ! \varepsilon / c^{k-1}\right\}, \\
W_{k}(c, s, \varepsilon)=\left\{\left(t, x, w_{0}, \ldots, w_{k-1}\right) \in \mathbb{C} \times \mathbb{C} \times \mathbb{C}^{k} ;|t| \leq \varepsilon,|x| \leq s,\right. & \left.\left|w_{0}\right| \leq 0 ! \varepsilon,\left|w_{1}\right| \leq 1 ! \varepsilon / c, \ldots,\left|w_{k-1}\right| \leq(k-1) ! \varepsilon / c^{k-1}\right\}
\end{aligned}
\end{aligned}
$$


$(k=1,2, \ldots)$. For a holomorphic function $f\left(t, x, u_{0}, \ldots, u_{k-1}\right)$ on $U_{k}=U_{k}(r$, $c, s, \varepsilon)$ we define the norm $\|f\|_{U_{k}}$ by

$$
\|f\|_{U_{k}}=\max _{U_{k}}\left|f\left(t, x, u_{0}, \ldots, u_{k-1}\right)\right| .
$$

The norm $\|g\|_{W_{k}}$ is defined in the same way. Then, by the results in subsections $3.1 \sim 3.4$ we have:

Theorem 3.5.2. $\quad$ Suppose the conditions $\left.\left.\left.\mathrm{A}_{1}\right), \mathrm{A}_{2}\right), \mathrm{A}_{3}\right)$ and that

$$
\begin{aligned}
& |i+\lambda(x)(j-1)| \geq \sigma_{o}(i+j) \quad \text { on } D_{R} \\
& \quad \text { for any }(i, j) \in \mathbb{N} \times \mathbb{N} \backslash\{(0,0),(0,1)\}
\end{aligned}
$$

for some $\sigma_{0}>0$. Then, we can find constants $r_{0}>0$ and $0<c_{0}<R$ so that the following results hold.

(1) The coupling equation $(\Phi)$ has a unique formal solution of the form

$$
\begin{aligned}
& \phi=\frac{-a(x)}{1-\lambda(x)} t+u_{0}+\sum_{k \geq 2} \phi_{k}\left(t, x, u_{0}, \ldots, u_{k-1}\right) \\
& \text { with } \phi_{k}\left(t, x, u_{0}, \ldots, u_{k-1}\right) \in \mathcal{H}_{k, R}\left[t, u_{0}, \ldots, u_{k-1}\right](k=2,3, \ldots) ;
\end{aligned}
$$

moreover, for any $0<r \leq r_{0}, 0<c \leq c_{0}$ and $s=R-c$ there is an $\varepsilon>0$ such that

$$
\sum_{k \geq 1}\left\|\phi_{k}\right\|_{U_{k}} \quad \text { with } U_{k}=U_{k}(r, c, s, \varepsilon)
$$

is convergent, where $\phi_{1}=-a(x) /(1-\lambda(x)) t+u_{0}$.

(2) The coupling equation $(\Psi)$ has a unique formal solution of the form

$$
\begin{aligned}
& \psi=\frac{a(x)}{1-\lambda(x)} t+w_{0}+\sum_{k \geq 2} \psi_{k}\left(t, x, w_{0}, \ldots, w_{k-1}\right) \\
& \text { with } \psi_{k}\left(t, x, w_{0}, \ldots, w_{k-1}\right) \in \mathcal{H}_{k, R}\left[t, w_{0}, \ldots, w_{k-1}\right](k=2,3, \ldots)
\end{aligned}
$$

moreover, for any $r>0,0<c \leq c_{0}$ and $0<s<R$ there is an $\varepsilon>0$ such that

$$
\sum_{k \geq 1}\left\|\psi_{k}\right\|_{W_{k}} \quad \text { with } W_{k}=W_{k}(c, s, \varepsilon)
$$

is convergent, where $\psi_{1}=a(x) /(1-\lambda(x)) t+w_{0}$.

(3) In addition, the relation $w=\phi(t, x, u, \partial u / \partial x, \ldots)$ is reversible with respect to $u$ and $w$, and the function $\psi\left(t, x, w_{0}, w_{1}, \ldots\right)$ is the reverse function of $\phi\left(t, x, u_{0}, u_{1}, \ldots\right)$. 
Thus, if we can find function-spaces $\mathcal{F}$ and $\mathcal{G}$ so that the two mappings

$$
\begin{aligned}
\Phi: \mathcal{F} \ni u(t, x) \longmapsto w(t, x) & =\phi(t, x, u, \partial u / \partial x, \ldots) \in \mathcal{G} \\
\Psi: \mathcal{G} \ni w(t, x) \longmapsto u(t, x) & =\psi(t, x, w, \partial w / \partial x, \ldots) \in \mathcal{F}
\end{aligned}
$$

are well-defined, we can conclude (by Theorem 2.6) that the two equations (3.5.1) and (3.5.2) are really equivalent. Let us define such function-spaces.

For simplicity, we denote by:

- $\mathcal{R}(\mathbb{C} \backslash\{0\})$ the universal covering space of $\mathbb{C} \backslash\{0\}$,

- $S_{\theta}=\{t \in \mathcal{R}(\mathbb{C} \backslash\{0\}) ;|\arg t|<\theta\}$ a sector in $\mathcal{R}(\mathbb{C} \backslash\{0\})$,

- $S_{\theta}(r)=\left\{t \in S_{\theta} ; 0<|t| \leq r\right\}$,

- $S(\varepsilon(s))=\{t \in \mathcal{R}(\mathbb{C} \backslash\{0\}) ; 0<|t|<\varepsilon(\arg t)\}$, where $\varepsilon(s)$ is a positive-valued continuous function on $\mathbb{R}_{s}$,

Definition 3.5.3. (1) We denote by $\widetilde{\mathcal{O}}_{+}$the set of all $u(t, x)$ satisfying the following i) and ii): i) $u(t, x)$ is a holomorphic function on $S(\varepsilon(s)) \times D_{R}$ for some positive-valued continuous function $\varepsilon(s)$ on $\mathbb{R}_{s}$ and $R>0$; and ii) there is an $a>0$ such that for any $\theta>0$ we have

$$
\max _{x \in D_{R}}|u(t, x)|=O\left(|t|^{a}\right) \quad\left(\text { as } t \longrightarrow 0 \text { in } S_{\theta}\right) .
$$

(2) We denote by $\mathscr{S}_{+}$the set of all $u(t, x)$ satisfying the following i) and ii): i) $u(t, x)$ is a holomorphic function on $S_{\theta}(r) \times D_{R}$ for some $\theta>0, r>0$ and $R>0$; and ii) there is an $a>0$ such that

$$
\max _{x \in D_{R}}|u(t, x)|=O\left(|t|^{a}\right) \quad\left(\text { as } t \longrightarrow 0 \text { in } S_{\theta}(r)\right) .
$$

Similarly we define

Definition 3.5.4. (1) We denote by $\widetilde{\mathcal{O}}_{\text {zero }}$ the set of all $u(t, x)$ satisfying the following i) and ii): i) $u(t, x)$ is a holomorphic function on $S(\varepsilon(s)) \times D_{R}$ for some positive-valued continuous function $\varepsilon(s)$ on $\mathbb{R}_{s}$ and $R>0$; and ii) for any $\theta>0$ we have

$$
\max _{x \in D_{R}}|u(t, x)|=o(1) \quad\left(\text { as } t \longrightarrow 0 \text { in } S_{\theta}\right) .
$$

(2) We denote by $\mathscr{S}_{\text {zero }}$ the set of all $u(t, x)$ satisfying the following i) and ii): i) $u(t, x)$ is a holomorphic function on $S_{\theta}(r) \times D_{R}$ for some $\theta>0, r>0$ and $R>0$; and ii) we have

$$
\max _{x \in D_{R}}|u(t, x)|=o(1) \quad\left(\text { as } t \longrightarrow 0 \text { in } S_{\theta}(r)\right) .
$$


Then, by Theorem 3.5.2 we can easily see:

Proposition 3.5.5. Set $\mathcal{F}=\widetilde{\mathcal{O}}_{+}, \mathscr{S}_{+}, \widetilde{\mathcal{O}}_{\text {zero }}$ or $\mathscr{S}_{\text {zero }}$. Then, the following two mappings are well-defined:

$$
\begin{aligned}
& \Phi: \mathcal{F} \ni u(t, x) \longmapsto w(t, x)=\phi(t, x, u, \partial u / \partial x, \ldots) \in \mathcal{F}, \\
& \Psi: \mathcal{F} \ni w(t, x) \longmapsto u(t, x)=\psi(t, x, w, \partial w / \partial x, \ldots) \in \mathcal{F} .
\end{aligned}
$$

Thus, we obtain

Theorem 3.5.6. Suppose the conditions $\left.\left.\mathrm{A}_{1}\right), \mathrm{A}_{2}\right), \mathrm{A}_{3}$ ) and that

$$
\begin{aligned}
& |i+\lambda(x)(j-1)| \geq \sigma_{o}(i+j) \quad \text { on } D_{R} \\
& \quad \text { for any }(i, j) \in \mathbb{N} \times \mathbb{N} \backslash\{(0,0),(0,1)\}
\end{aligned}
$$

for some $\sigma_{0}>0$. Set $\mathcal{F}=\widetilde{\mathcal{O}}_{+}, \mathscr{S}_{+}, \widetilde{\mathcal{O}}_{\text {zero }}$ or $\mathscr{S}_{\text {zero }}$. Then, the following two equations are equivalent:

$$
\begin{aligned}
& t \frac{\partial u}{\partial t}=F\left(t, x, u, \frac{\partial u}{\partial x}\right) \quad \text { in } \mathcal{F}, \\
& t \frac{\partial w}{\partial t}=\lambda(x) w \quad \text { in } \mathcal{F} .
\end{aligned}
$$

\section{$\S 4 . \quad$ Application}

In this section, by using the equivalence theorem (Theorem 3.5.6) we will investigate the structure of solutions of Briot-Bouquet type partial differential equation

$$
t \frac{\partial u}{\partial t}=F\left(t, x, u, \frac{\partial u}{\partial x}\right)
$$

under the same assumptions $\left.A_{1}\right), A_{2}$ ) and $A_{3}$ ).

\section{$\S 4.1$. Solutions in a sectorial domain}

First, we note:

Lemma 4.1. The following two conditions are equivalent:

(1) $\lambda(0) \notin(-\infty, 0] \cup\{1,2, \ldots\}$.

(2) There are $\sigma_{0}>0$ and $R>0$ such that $|i+\lambda(x)(j-1)| \geq \sigma_{0}(i+j)$ holds on $D_{R}$ for any $(i, j) \in \mathbb{N}^{2} \backslash\{(0,0),(0,1)\}$. 
Proof. Denote by $L(1, \lambda(x))$ the segment in the complex plane joining the two points 1 and $\lambda(x)$. For $(i, j) \in \mathbb{N}^{2} \backslash\{(0,0),(0,1)\}$ we set

$$
z_{i, j}(x)=\frac{i+\lambda(x) j}{i+j}
$$

which is the point on $L(1, \lambda(x))$ such that $\left|1-z_{i, j}(x)\right|:\left|z_{i, j}(x)-\lambda(x)\right|=j: i$.

Let us show that (1) implies (2). Suppose the condition (1); then we have $\lambda(x) \notin(-\infty, 0] \cup\{1,2, \ldots\}$ on $D_{R}$ for some $R>0$, and so $|i+\lambda(x)(j-1)| \neq 0$ for any $(i, j) \in \mathbb{N}^{2} \backslash\{(0,0),(0,1)\}$ and $x \in D_{R}$. Moreover, if we denote by $d(x)$ the distance from the origin to $L(1, \lambda(x))$, we have $d(x) \geq c$ on $D_{R}$ for some $c>0$; this implies $\left|z_{i, j}(x)\right| \geq c$ for any $x \in D_{R}$.

Choose a sufficiently large $N$ so that $|\lambda(x)| / N \leq c / 2$ on $D_{R}$. Then, if $(i, j) \in \mathbb{N}^{2}$ satisfies $i+j \geq N$ we see

$$
\left|\frac{i+\lambda(x)(j-1)}{i+j}\right| \geq\left|z_{i, j}(x)\right|-\frac{|\lambda(x)|}{i+j} \geq c-\frac{|\lambda(x)|}{N} \geq c-\frac{c}{2} \geq \frac{c}{2} \quad \text { on } D_{R} .
$$

On the other hand, since the number of the elements of $I_{N}=\left\{(i, j) \in \mathbb{N}^{2} \backslash\right.$ $\{(0,0),(0,1)\} ; i+j<N\}$ is finite, and since $|i+\lambda(x)(j-1)| \neq 0$ for any $(i, j) \in I_{N}$ and $x \in D_{R}$ we have

$$
|i+\lambda(x)(j-1)| \geq \sigma(i+j) \quad \text { on } D_{R} \text { for any }(i, j) \in I_{N}
$$

for some $\sigma>0$. Thus, by setting $\sigma_{0}=\min \{c / 2, \sigma\}$ we have the condition (2).

Next let us show that (2) implies (1). Suppose the condition (2). Then, by considering the case $j=0$ and $x=0$ we have $|i-\lambda(0)| \geq \sigma_{0} i$ for any $i \in\{1,2, \ldots\}$. This implies $\lambda(0) \notin\{1,2, \ldots\}$.

The condition $\lambda(0) \notin(-\infty, 0]$ is verified as follows. Choose a sufficiently large $N$ so that $|\lambda(0)| / N \leq \sigma_{0} / 2$. Then, if $(i, j) \in \mathbb{N}^{2}$ satisfies $i+j \geq N$ we have

$$
\left|z_{i, j}(0)\right| \geq\left|\frac{i+\lambda(0)(j-1)}{i+j}\right|-\frac{|\lambda(0)|}{i+j} \geq \sigma_{0}-\frac{|\lambda(0)|}{N} \geq \sigma_{0}-\frac{\sigma_{0}}{2} \geq \frac{\sigma_{0}}{2} .
$$

This implies that the distance from the origin to $L(1, \lambda(0))$ is greater than or equal to $\sigma_{0} / 2$, because the set $\left\{z_{i, j}(0) ;(i, j) \in \mathbb{N}^{2}\right.$ and $\left.i+j \geq N\right\}$ is dense in $L(1, \lambda(0))$. Thus, we obtain the condition that $L(1, \lambda(0))$ does not contain the origin and therefore $\lambda(0) \notin(-\infty, 0]$.

Thus, by this lemma we see that all the results in section 3 are valid under the condition

$$
\lambda(0) \notin(-\infty, 0] \cup\{1,2, \ldots\} .
$$


Now, let us determine all the singular solutions of the equation (4.1). For a function-class $\mathcal{F}$ we set:

$$
\operatorname{Sol}(\mathcal{F})=\text { the set of all solutions of }(4.1) \text { in } \mathcal{F} .
$$

Let $\psi\left(t, x, w_{0}, w_{1}, \ldots\right)$ be the solution of $(\Psi)$ in $(2)$ of Theorem 3.5.2, and we write:

$$
\Psi[w]=\psi(t, x, w, \partial w / \partial x, \ldots) .
$$

We denote by $\mathbb{C}\{x\}$ the ring of convergent power series in $x$ which is the set of all holomorphic functions in a neighborhood of $x=0 \in \mathbb{C}$. We have

Theorem 4.2. $\quad$ Suppose the conditions $\left.\left.\left.\mathrm{A}_{1}\right), \mathrm{A}_{2}\right), \mathrm{A}_{3}\right)$ and (4.2). Then, we have the following result.

(1) If $\operatorname{Re} \lambda(0)>0$, we have

$$
\begin{aligned}
\operatorname{Sol}\left(\widetilde{\mathcal{O}}_{+}\right) & =\operatorname{Sol}\left(\mathscr{S}_{+}\right)=\operatorname{Sol}\left(\widetilde{\mathcal{O}}_{\text {zero }}\right)=\operatorname{Sol}\left(\mathscr{S}_{\text {zero }}\right) \\
& =\left\{\Psi\left[h(x) t^{\lambda(x)}\right] ; h(x) \in \mathbb{C}\{x\}\right\} .
\end{aligned}
$$

(2) If $\operatorname{Re} \lambda(0) \leq 0$, we have

$$
\operatorname{Sol}\left(\widetilde{\mathcal{O}}_{+}\right)=\operatorname{Sol}\left(\mathscr{S}_{+}\right)=\operatorname{Sol}\left(\widetilde{\mathcal{O}}_{\text {zero }}\right)=\operatorname{Sol}\left(\mathscr{S}_{\text {zero }}\right)=\{\Psi[0]\} .
$$

Note that $\Psi[0]=\psi(t, x, 0,0, \ldots)\left(=u_{0}(t, x)\right)$ is the unique holomorphic solution satisfying $u_{0}(0, x)=0$ (obtained in Theorem 1.1).

Proof. By Theorem 3.5.6 it is sufficient to solve the equation

$$
t \frac{\partial w}{\partial t}=\lambda(x) w \text { in } \mathcal{F}
$$

with $\mathcal{F}=\widetilde{\mathcal{O}}_{+}, \mathscr{S}_{+}, \widetilde{\mathcal{O}}_{\text {zero }}$ or $\mathscr{S}_{\text {zero }}$.

Let us denote by $\mathscr{S}_{*}$ the set of all holomorphic functions $u(t, x)$ on $S_{\theta}(r) \times$ $D_{R}$ for some $\theta>0, r>0$ and $R>0$ (which may depend on $u(t, x)$ ). It is easy to see that the set $\operatorname{Sol}\left((4.5), \mathscr{S}_{*}\right)$ of all the solutions of this equation (4.5) with $\mathcal{F}=\mathscr{S}_{*}$ is given by

$$
\operatorname{Sol}\left((4.5), \mathscr{S}_{*}\right)=\left\{h(x) t^{\lambda(x)} ; h(x) \in \mathbb{C}\{x\}\right\} .
$$

Therefore, Theorem 4.2 follows from Lemma 4.3 given below and the following fact:

$$
\widetilde{\mathcal{O}}_{+} \subset \mathscr{S}_{+} \subset \mathscr{S}_{\text {zero }} \subset \mathscr{S}_{*}, \quad \text { and } \quad \widetilde{\mathcal{O}}_{+} \subset \widetilde{\mathcal{O}}_{\text {zero }} \subset \mathscr{S}_{\text {zero }} \subset \mathscr{S}_{*} \text {. }
$$


Lemma 4.3. (1) If $\operatorname{Re} \lambda(0)>0$ and $h(x) \in \mathbb{C}\{x\}$, there are $a>0$ and $R>0$ such that

$$
\max _{D_{R}}\left|h(x) t^{\lambda(x)}\right|=O\left(|t|^{a}\right) \quad\left(\text { as } t \longrightarrow 0 \text { in } S_{\theta}\right)
$$

holds for any $\theta>0$.

(2) If $\operatorname{Re} \lambda(0) \leq 0$ and

$$
\max _{D_{R}}\left|h(x) t^{\lambda(x)}\right|=o(1) \quad\left(\text { as } t \longrightarrow 0 \text { in } S_{\theta}\right)
$$

for some $R>0$ and $\theta>0$, we have $h(x) \equiv 0$.

Proof of Lemma 4.3. If $\operatorname{Re} \lambda(0)>0$, we have $\operatorname{Re} \lambda(x) \geq a$ on $D_{R}$ for some $a>0$ and $R>0$; then we can easily see the condition (4.6).

Let us show (2). If $\operatorname{Re} \lambda(0)<0$, we may assume that $\operatorname{Re} \lambda(x)<-a$ on $D_{R}$ for some $a>0$, and then by (4.7) we have

$$
\max _{D_{R}}|h(x)| \leq \max _{D_{R}}\left|h(x) t^{\lambda(x)}\right| \times \max _{D_{R}}\left|t^{-\lambda(x)}\right|=o(1) \times O\left(|t|^{a}\right)
$$

(as $t \longrightarrow 0$ in $S_{\theta}$ ): this proves $h(x) \equiv 0$. If $\operatorname{Re} \lambda(0)=0$ and $\operatorname{Re} \lambda(x) \not \equiv 0$, we can find an $x_{0} \in D_{R}$ such that $\operatorname{Re} \lambda\left(x_{0}\right)<0$, and so by the same argument as above we have $h(x) \equiv 0$ in a neighborhood of $x=x_{0}$; this proves $h(x) \equiv 0$. If $\operatorname{Re} \lambda(x) \equiv 0$, we have $\left|t^{-\lambda(x)}\right|=O(1)$ (as $t \longrightarrow 0$ in $S_{\theta}$ ) and so by (4.7) we have

$$
\max _{D_{R}}|h(x)| \leq \max _{D_{R}}\left|h(x) t^{\lambda(x)}\right| \times \max _{D_{R}}\left|t^{-\lambda(x)}\right|=o(1) \times O(1)
$$

(as $t \longrightarrow 0$ in $S_{\theta}$ ): this proves $h(x) \equiv 0$.

By this lemma we see: (1) if $\operatorname{Re} \lambda(0)>0$ we have $h(x) t^{\lambda(x)} \in \widetilde{\mathcal{O}}_{+}$for any $h(x) \in \mathbb{C}\{x\}$, and (2) if $\operatorname{Re} \lambda(0) \leq 0$ and $h(x) t^{\lambda(x)} \in \mathscr{S}_{\text {zero }}$ hold we have $h(x) \equiv 0$. This completes the proof of Theorem 4.2.

Since $\Psi[0]=\psi(t, x, 0,0, \ldots)$ is nothing but the unique holomorphic solution $u_{0}(t, x)$ obtained in Theorem 1.1, we have

Theorem 4.4 (Analytic continuation). Suppose the conditions $\left.\mathrm{A}_{1}\right), \mathrm{A}_{2}$ ), $\left.\mathrm{A}_{3}\right), \operatorname{Re} \lambda(0) \leq 0$ and $\lambda(0) \notin(-\infty, 0]$. If $u(t, x)$ is a holomorphic solution of (4.1) on $S_{\theta}(r) \times D_{R}$ satisfying $u(t, x) \longrightarrow 0$ uniformly on $D_{R}$ (as $t \longrightarrow 0$ in $\left.S_{\theta}(r)\right)$, then $u(t, x)$ has an analytic continuation up to some neighborhood of $(0,0) \in \mathbb{C}_{t} \times \mathbb{C}_{x}$ 
Remark. The author believes that the analytic continuation in Theorem 4.4 is valid also in the case $\lambda(0) \in(-\infty, 0)$. But, to prove this we need a completely different proof, because the condition $\lambda(0) \notin(-\infty, 0]$ is the main part of our Poincaré condition (3.5.3) and it is essential to our transformation theory. In the case $\lambda(0)=0$, we have the following example: the equation $t(\partial u / \partial t)=u(\partial u / \partial x)$ has a family of solutions $u=(x+c) /(-\log t)$ with $c \in \mathbb{C}$.

Now, let us see the concrete form of the solution $\Psi\left[h(x) t^{\lambda(x)}\right]$. By Proposition 3.2.3 we see that $\psi\left(t, x, w_{0}, w_{1}, \ldots\right)$ has the form

$$
\begin{aligned}
\psi=\frac{a(x)}{1-\lambda(x)} t & +w_{0}+\sum_{k \geq 2}\left[\psi_{k, 0}(x) t^{k}\right. \\
& \left.+\sum_{i+|j|=k,|j| \geq 1, i+2(|j|-1) \geq\langle j\rangle} \psi_{i, j}(x) t^{i} w_{0}{ }^{j_{0}} \cdots w_{k-1}{ }^{j_{k-1}}\right],
\end{aligned}
$$

and so we have

$$
\begin{gathered}
\Psi\left[h(x) t^{\lambda(x)}\right]=\frac{a(x)}{1-\lambda(x)} t+h(x) t^{\lambda(x)}+\sum_{k \geq 2} \psi_{k, 0}(x) t^{k} \\
+\sum_{\substack{i+|j| \geq 2,|j| \geq 1 \\
i+2(|j|-1) \geq\langle j\rangle}} \psi_{i, j}(x) t^{i}\left[h(x) t^{\lambda(x)}\right]^{j_{0}}\left[\left(\frac{\partial}{\partial x}\right)\left(h(x) t^{\lambda(x)}\right)\right]^{j_{1}} \times \\
\times \cdots \times\left[\left(\frac{\partial}{\partial x}\right)^{k-1}\left(h(x) t^{\lambda(x)}\right)\right]^{j_{k-1}} .
\end{gathered}
$$

Since $(\partial / \partial x)^{i}\left(h(x) t^{\lambda(x)}\right)$ is expressed in the form

$$
\left(\frac{\partial}{\partial x}\right)^{i}\left(h(x) t^{\lambda(x)}\right)=\sum_{0 \leq p \leq i} h_{p}(x) t^{\lambda(x)}(\log t)^{p}, \quad 0 \leq i \leq k-1,
$$

by substituting this into (4.8) we have the following result:

Proposition 4.5. In the case $\operatorname{Re} \lambda(0)>0$, the $\widetilde{\mathcal{O}}_{+}$-solution $\Psi\left[h(x) t^{\lambda(x)}\right]$ (with $h(x) \in \mathbb{C}\{x\}$ ) has the expansion of the form

$$
\begin{aligned}
\Psi\left[h(x) t^{\lambda(x)}\right]= & \frac{a(x)}{1-\lambda(x)} t+\sum_{k \geq 2} \psi_{k, 0}(x) t^{k} \\
& +h(x) t^{\lambda(x)}+\sum_{\substack{i+j \geq 2, j \geq 1 \\
i+2(j-1) \geq p}} h_{i, j, p}(x) t^{i+j \lambda(x)}(\log t)^{p}
\end{aligned}
$$

for some holomorphic functions $\psi_{k, 0}(x)(k \geq 2)$ and $h_{i, j, p}(x)(i+j \geq 2, j \geq 1$ and $i+2(j-1) \geq p)$ on $D_{R}$. 
The case $\mathcal{F}=\widetilde{\mathcal{O}}_{+}$of Theorem 4.2 (and Proposition 4.5) is already written in the book of Gérard-Tahara [1] (chapter 5): the case $\mathcal{F}=\mathscr{S}_{+}$is not written explicitly in [1], but one can easily see that the proof in [1] works also in the case $\mathcal{F}=\mathscr{S}_{+}$. Recall:

Theorem 4.6 (Theorem 5.2.3 in [1]). Suppose the conditions $\mathrm{A}_{1}$ ), $\mathrm{A}_{2}$ ), $\left.\mathrm{A}_{3}\right)$ and $\lambda(0) \notin\{1,2, \ldots\}$. Then we have the following results.

(1) The equation (4.1) has a unique holomorphic solution $u_{0}(t, x)$ in a neighborhood of the origin of $\mathbb{C}_{t} \times \mathbb{C}_{x}$ satisfying $u_{0}(0, x) \equiv 0$.

(2) We denote by $\operatorname{Sol}\left(\widetilde{\mathcal{O}}_{+}\right)\left(\operatorname{resp} . \operatorname{Sol}\left(\mathscr{S}_{+}\right)\right)$the set of all the solutions of (4.1) belonging in the class $\widetilde{\mathcal{O}}_{+}\left(\right.$resp. $\left.\mathscr{S}_{+}\right)$. We have:

(2-1) If $\operatorname{Re} \lambda(0) \leq 0$, we have

$$
\operatorname{Sol}\left(\widetilde{\mathcal{O}}_{+}\right)=\operatorname{Sol}\left(\mathscr{S}_{+}\right)=\left\{u_{0}(t, x)\right\},
$$

where $u_{0}(t, x)$ is the unique holomorphic solution obtained in (1).

(2-2) If $\operatorname{Re} \lambda(0)>0$, we have

$$
\operatorname{Sol}\left(\widetilde{\mathcal{O}}_{+}\right)=\operatorname{Sol}\left(\mathscr{S}_{+}\right)=\left\{u_{0}(t, x)\right\} \cup\{U(\varphi) ; 0 \neq \varphi(x) \in \mathbb{C}\{x\}\},
$$

where $U(\varphi)$ is an $\widetilde{\mathcal{O}}_{+}$-solution of (4.1) having the expansion of the following form:

$$
U(\varphi)=\sum_{i \geq 1} u_{i}(x) t^{i}+\sum_{i+2 j \geq p+2, j \geq 1} \varphi_{i, j, p}(x) t^{i+j \lambda(x)}(\log t)^{p}
$$

with $\varphi_{0,1,0}(x)=\varphi(x)$.

\section{$\S 4.2$. Solutions in a spiral domain}

First, let us illustrate what we are thinking from now.

Example 4.7. Let us consider the equation

$$
t \frac{d u}{d t}=(-1+i) u \quad(\text { where } i=\sqrt{-1}) .
$$

We have a general solution $u(t)=A t^{-1+i}$ with an arbitrary constant $A \in \mathbb{C}$.

(1) As is seen in Lemma 4.3, if $A t^{-1+i}=o(1)$ (as $t \longrightarrow 0$ in $S_{\theta}$ ) we have $A=0$ : therefore, if $u(t)$ is a solution of (4.12) belonging in the class $\mathscr{S}_{\text {zero }}$ we have $u(t) \equiv 0$.

(2) But, if we consider the curve $C=\left\{t \in \mathcal{R}\left(\mathbb{C}_{t} \backslash\{0\}\right) ; 2 \log |t|+\arg t=0\right\}$ we have $A t^{-1+i}=o(1)$ (as $C \ni t \longrightarrow 0$ ) for any $A \in \mathbb{C}$. This is verified as follows. Set $t=r e^{i \phi}$; then we have

$$
\left|t^{-1+i}\right|=\left|\left(r e^{i \phi}\right)^{-1+i}\right|=r^{-1} e^{-\phi}=e^{-\log r-\phi}
$$


therefore $t^{-1+i} \longrightarrow 0$ is equivalent to the condition $\log r+\phi \longrightarrow \infty$. Since $C$ is expressed as $C=\left\{t=r e^{i \phi} ; 2 \log r+\phi=0\right\}$, the condition $C \ni t \longrightarrow 0$ implies that $\log r \longrightarrow-\infty$ and so $\log r+\phi=-\log r \longrightarrow \infty$ hold. This proves that $t^{-1+i} \longrightarrow 0$ (as $\left.C \ni t \longrightarrow 0\right)$.

(3) Thus, if we set

$$
\begin{aligned}
& C=\left\{t \in \mathcal{R}\left(\mathbb{C}_{t} \backslash\{0\}\right) ; 2 \log |t|+\arg t=0\right\}, \\
& \Omega=\left\{t \in \mathcal{R}\left(\mathbb{C}_{t} \backslash\{0\}\right) ;-1<2 \log |t|+\arg t<1\right\}
\end{aligned}
$$

we have the properties: i) $C$ is a curve such that $t(\in C)$ can approach the origin along the curve $C$, ii) $\Omega$ is a neighborhood of $C$ on which $A t^{-1+i}$ is a solution of (4.12), and iii) $A t^{-1+i} \longrightarrow 0$ (as $C \ni t \longrightarrow 0$ ).

Motivated by this illustration, we will define:

Definition 4.8. We denote by $\mathscr{C}_{\text {zero }}$ the set of all $u(t, x)$ such that there are a curve $C$ in $\mathcal{R}(\mathbb{C} \backslash\{0\})$, a neighborhood $\Omega$ of $C$, and $R>0$ which satisfy the following properties: i) $t(\in C)$ can approach the origin along the curve $C$, ii) $u(t, x)$ is a holomorphic function on $\Omega \times D_{R}$, and iii) we have

$$
\max _{x \in D_{R}}|u(t, x)|=o(1) \quad(\text { as } C \ni t \longrightarrow 0) .
$$

(Note that $C, \Omega$ and $R$ may depend on $u(t, x)$.)

Then, we have

Theorem 4.9. Suppose the conditions $\left.\left.\left.\mathrm{A}_{1}\right), \mathrm{A}_{2}\right), \mathrm{A}_{3}\right)$ and (4.2). Then, the following two equations are equivalent:

$$
\begin{aligned}
t \frac{\partial u}{\partial t} & =F\left(t, x, u, \frac{\partial u}{\partial x}\right) \quad \text { in } \mathscr{C}_{z e r o}, \\
t \frac{\partial w}{\partial t} & =\lambda(x) w \quad \text { in } \mathscr{C}_{z e r o} .
\end{aligned}
$$

As an application we have

Theorem 4.10. Suppose the conditions $\left.\left.\left.\mathrm{A}_{1}\right), \mathrm{A}_{2}\right), \mathrm{A}_{3}\right)$ and (4.2). Denote by Sol $\left(\mathscr{C}_{\text {zero }}\right)$ the set of all the solutions of (4.1) belonging in the class $\mathscr{C}_{\text {zero }}$. Then we have

$$
\operatorname{Sol}\left(\mathscr{C}_{\text {zero }}\right)=\left\{\Phi\left[h(x) t^{\lambda(x)}\right] ; h(x) \in \mathbb{C}\{x\}\right\} .
$$

This follows from 
Lemma 4.11. If $\lambda(0) \notin(-\infty, 0]$, we can find a curve $C$ in $\mathcal{R}(\mathbb{C} \backslash\{0\})$ such that $t(\in C)$ can approach the origin along the curve $C$ and that

$$
\max _{x \in D_{R}}\left|t^{\lambda(x)}\right|=o(1) \quad(\text { as } C \ni t \longrightarrow 0) .
$$

Proof. If $\operatorname{Re} \lambda(0)>0$, by taking $C=\{t \in \mathbb{R} ; 0<t<\delta\}$ for a sufficiently small $\delta>0$ we have (4.15). If $\operatorname{Re} \lambda(0) \leq 0$ and $\operatorname{Im} \lambda(0)>0$, then we can find $a>0, b>0$ and $R>0$ such that $|\operatorname{Re} \lambda(x)| \leq a$ and $\operatorname{Im} \lambda(x)>b$ on $D_{R}$ hold; then for any $t=r e^{i \phi}$ (with $\phi>0$ ) we have

$$
\max _{x \in D_{R}}\left|t^{\lambda(x)}\right| \leq r^{-a} e^{-b \phi}=e^{-a \log r-b \phi}
$$

and so by setting $C=\left\{t=r e^{i \phi} ; 2 a \log r+b \phi=0, \phi>0\right\}$ we have the condition (4.15). If $\operatorname{Re} \lambda(0) \leq 0$ and $\operatorname{Im} \lambda(0)<0$, we can prove (4.15) in the same way.

Proof of Theorem 4.10. Since Theorem 4.9 is already known, we have only to notice the following facts: (i) in the case $|\operatorname{Re} \lambda(x)| \leq a$ and $\operatorname{Im} \lambda(x)>b$ on $D_{R}$ (with $a>0, b>0$ and $R>0$ ), the function $h(x) t^{\lambda(x)}$ is a solution of (4.14) in a spiral domain $\Omega=\left\{(t, x) \in \mathcal{R}\left(\mathbb{C}_{t} \backslash\{0\}\right) \times D_{R} ;-1<2 a \log |t|+\right.$ $b \arg t<1, \log |t|<-A\}$ for some $A>0$, and (ii) in the case $|\operatorname{Re} \lambda(x)| \leq a$ and $\operatorname{Im} \lambda(x)<-b$ on $D_{R}$ (with $a>0, b>0$ and $R>0$ ), the function $h(x) t^{\lambda(x)}$ is a solution of (4.14) in a spiral domain $\Omega=\left\{(t, x) \in \mathcal{R}\left(\mathbb{C}_{t} \backslash\{0\}\right) \times D_{R} ;-1<\right.$ $2 a \log |t|-b \arg t<1, \log |t|<-A\}$ for some $A>0$.

Similarly to Proposition 4.5 we have

Proposition 4.12. In the case $\operatorname{Re} \lambda(0) \leq 0$ and $\lambda(0) \notin(-\infty, 0]$, the function $\Psi\left[h(x) t^{\lambda(x)}\right]$ (with $h(x) \in \mathbb{C}\{x\}$ ) defines a holomorphic solution of (4.1) in a spiral domain $\Omega_{+}=\left\{(t, x) \in \mathcal{R}\left(\mathbb{C}_{t} \backslash\{0\}\right) \times D_{R} ;-1<2 a \log |t|+\right.$ $b \arg t<1, \log |t|<-A\}$ or $\Omega_{-}=\left\{(t, x) \in \mathcal{R}\left(\mathbb{C}_{t} \backslash\{0\}\right) \times D_{R} ;-1<2 a \log |t|-\right.$ $b \arg t<1, \log |t|<-A\}$ for some $R>0, a>0, b>0$ and $A>0$, and it has the expansion of the form

$$
\begin{aligned}
\Psi\left[h(x) t^{\lambda(x)}\right]= & \frac{a(x)}{1-\lambda(x)} t+\sum_{k \geq 2} \psi_{k, 0}(x) t^{k} \\
& +h(x) t^{\lambda(x)}+\sum_{\substack{i+j \geq 2, j \geq 1 \\
i+2(j-1) \geq p}} h_{i, j, p}(x) t^{i+j \lambda(x)}(\log t)^{p}
\end{aligned}
$$

for some holomorphic functions $\psi_{k, 0}(x)(k \geq 2)$ and $h_{i, j, p}(x)(i+j \geq 2, j \geq 1$ and $i+2(j-1) \geq p)$ on $D_{R}$. 


\section{References}

[1] R. Gérard and H. Tahara, Singular nonlinear partial differential equations, Aspects of Mathematics. Friedr., Vieweg, Braunschweig, 1996.

[2] Holomorphic and singular solutions of nonlinear singular first order partial differential equations, Publ. Res. Inst. Math. Sci. 26 (1990), no. 6, 979-1000.

[3] H. Tahara, Coupling of two partial differential equations and its application, Publ. Res. Inst. Math. Sci. 43 (2007), no. 3, 535-583.

[4] H. Yamazawa, Singular solutions of the Briot-Bouquet type partial differential equations, J. Math. Soc. Japan 55 (2003), no. 3, 617-632. 